\title{
ON EXPLAINABLE FUZZY RECOMMENDERS AND THEIR PERFORMANCE EVALUATION
}

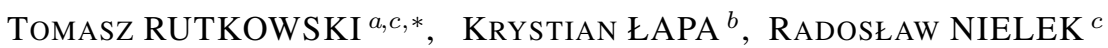 \\ ${ }^{a}$ Senfino, 1412 Broadway 21st floor, New York City, NY 10018, USA \\ e-mail: XAI@senfino.com \\ ${ }^{b}$ Institute of Computational Intelligence \\ Częstochowa University of Technology, Al. Armii Krajowej 36, 42-200 Częstochowa, Poland \\ ${ }^{c}$ Faculty of Information Technology \\ Polish-Japanese Academy of Information Technology, Koszykowa 86, 02-008 Warsaw, Poland
}

\begin{abstract}
This paper presents a novel approach to the design of explainable recommender systems. It is based on the Wang-Mendel algorithm of fuzzy rule generation. A method for the learning and reduction of the fuzzy recommender is proposed along with feature encoding. Three criteria, including the Akaike information criterion, are used for evaluating an optimal balance between recommender accuracy and interpretability. Simulation results verify the effectiveness of the presented recommender system and illustrate its performance on the MovieLens 10M dataset.
\end{abstract}

Keywords: recommender systems, explainable recommendations, fuzzy systems, Akaike information criterion.

\section{Introduction}

In the literature, several types of recommender systems have been presented (see, e.g., Lops et al., 2011; Wei et al., 2017; Zhang et al., 2018), and the most popular techniques are known under the names "content-based filtering" and "collaborative filtering."

In the case of content-based filtering, recommender systems suggest to a user items (e.g., movies or books) characterized by features similar to those ones that the user preferred in the past. In this scenario, recommendations are based on the content of a given item. In collaborative filtering, items are recommended to a user by similarities to other users (similar users' preferences). In that scenario, recommendations are based on other users' rates concerning the items and, e.g., the weighted average of the rates.

Modern recommender systems are designed with the use of machine learning algorithms. For an excellent survey, a reader is referred to the work of Portugal et al. (2018). The major drawback of the existing techniques is the lack of explainability. In many applications,

\footnotetext{
*Corresponding author
}

e.g., medical diagnosis or venture capital investment recommendations, it is essential to explain the rationale behind a specific recommendation. Motivated by this fact, in this paper we propose a novel approach to design explainable recommenders.

In order to create an explainable recommendation system, fuzzy IF-THEN rules are employed to represent knowledge about users' preferences. Then, the inference based on fuzzy logic is applied in the Mamdani fuzzy system (see, e.g., Rutkowska, 2002) to produce the recommendations. The method of fuzzy rule generation, proposed by Wang and Mendel (1991), is now combined with a technique of rule reduction. This approach is enhanced by three criteria, including the Akaike information criterion (see, e.g., Söderström and Stoica, 1989), which allow evaluating an optimal balance in terms of the recommender's accuracy and interpretability.

The explainability of the proposed recommender is assured due to the following:

1. Interpretable fuzzy rules with fuzzy sets as linguistic values of attributes describing items and users' preferences (fuzzy sets with semantic meanings). 
2. Incorporation of rule weights into the fuzzy system. The weights can be interpreted with regard to rule importance.

3. The reduction of the fuzzy rules makes the rule base simpler, and thus easier to produce explainable recommenders.

It is worth emphasizing that this approach leads to a moderate number of interpretable fuzzy rules and, in consequence, greatly facilities the explanation of the recommender system.

Moreover, the use of the Akaike information criterion, as well as the final prediction error and the Schwartz criterion (see Section 3.3), allows solving the problem of the compromise between the system error and the number of rules.

The presented approach greatly improves our previous attempts (Rutkowski et al., 2018a; 2018b) to the design of explainable recommender systems. The paper is organized as follows. Section 2 presents a short description of the Wang-Mendel rule generation method for construction of the Mamdani system with rule weighting factors. This is the background and our starting point to develop explainable fuzzy recommenders. The main part of this contribution is given in Section 3 by describing the proposed method (Algorithm 1) and feature encoding along with model evaluation criteria. Simulation results illustrating the performance of the system are described in Section 4 . Finally, Section 5 contains the main conclusions and remarks.

\section{Interpretable fuzzy rules}

In order to employ fuzzy IF-THEN rules in the recommender system proposed in this paper, we recall the idea of the Wang-Mendel algorithm (see Wang and Mendel, 1991) for fuzzy rule generation.

It should be emphasized that the material which we present below is perhaps the first mathematically precise description of that method in the multidimensional case. Now, we introduce the notation that will be useful in describing a recommendation system and its features' encoding (see Section 3.2).

We consider a fuzzy system with $n$ inputs and one output. Let $x_{1}, x_{2}, \ldots, x_{n}$ and $y$ be linguistic variables corresponding to input and output variables, respectively, of the fuzzy system.

The input vector $\mathbf{x}=\left[x_{1}, x_{2}, \ldots, x_{n}\right]^{T}$ in the space $\mathbf{X}=\mathbf{X}_{1} \times \mathbf{X}_{2} \times \cdots \times \mathbf{X}_{n}$, as well as $y \in Y$, can take crisp values, denoted as $\overline{\mathbf{x}}=\left[\bar{x}_{1}, \bar{x}_{2}, \ldots, \bar{x}_{n}\right]^{T}$ and $\bar{y}$, respectively; in this case, each universe of discourse can be the space of real numbers.

With regard to fuzzy sets in fuzzy IF-THEN rules, it should be noted that generally different numbers of linguistic values (fuzzy sets) can be defined for particular linguistic variables. Denote by $N_{i}$ the number of linguistic fuzzy sets for $x_{i}, i=1,2, \ldots, n$, and by $N_{y}$ the number of fuzzy sets for $y$.

Assume that the input fuzzy sets, denoted by $A_{i l}$, are defined by membership functions $\mu_{A_{i l}}\left(x_{i}\right), i=$ $1,2, \ldots, n$, and $l=1,2, \ldots, N_{i}$, in the universes of discourse $X_{1}, X_{2}, \ldots, X_{n}$, and analogously the output fuzzy sets, $B_{l}$, are defined by membership functions $\mu_{B_{l}}(y), l=1,2, \ldots, N_{y}$, in $Y$.

We can assign linguistic values for the inputs and output as follows:

$$
\begin{gathered}
x_{1}: A_{11}, A_{12}, \ldots, A_{1 N_{1}}, \\
x_{2}: A_{21}, A_{22}, \ldots, A_{2 N_{2}}, \\
\vdots \\
x_{n}: A_{n 1}, A_{n 2}, \ldots, A_{n N_{n}}, \\
y: B_{1}, B_{2}, \ldots, B_{N_{y}} .
\end{gathered}
$$

Suppose that we recorded $M$ input-output data pairs $\overline{\mathbf{x}}^{(j)}, \bar{y}^{(j)}$, where $j=1,2, \ldots, M$, and $\overline{\mathbf{x}}^{(j)}=$ $\left[\bar{x}_{1}{ }^{(j)}, \bar{x}_{2}{ }^{(j)}, \ldots, \bar{x}_{n}{ }^{(j)}\right]^{T}$.

The task is to generate a set of fuzzy IF-THEN rules from the input-output data. The first step of the Wang-Mendel method is to divide the input and output spaces into fuzzy regions. Then, in the second step, fuzzy rules are generated from given data pairs. The number of fuzzy regions refers to the numbers of linguistic values, $N_{i}$, for $i=1,2, \ldots, n$, and $N_{y}$.

Having $M$ data pairs $\overline{\mathbf{x}}^{(j)}, y^{(j)}$, for $j=1,2, \ldots, M$, degrees of membership in different regions are determined, and every data pair is put into the region of maximal degree, according to the equations

$$
\mu_{A_{i}^{j}}\left(\bar{x}_{i}^{(j)}\right)=\max _{l=1,2, \ldots, N_{i}}\left\{\mu_{A_{i l}}\left(\bar{x}_{i}{ }^{(j)}\right)\right\},
$$

for $i=1,2, \ldots, n$, and

$$
\mu_{B^{j}}\left(\bar{y}^{(j)}\right)=\max _{l=1,2, \ldots, N_{y}}\left\{\mu_{B_{l}}\left(\bar{y}^{(j)}\right)\right\}
$$

Finally, we obtain one rule from one input-output data pair. In this way, from the second step, we get $M$ fuzzy IF-THEN rules, $R^{j}$, for $j=1,2, \ldots, M$, of the following form:

$$
\begin{aligned}
& \text { IF } x_{1} \text { is } A_{1}^{j} \text { AND } x_{2} \text { is } A_{2}^{j} \operatorname{AND} \ldots \text { AND } x_{n} \text { is } A_{n}^{j} \\
& \text { THEN } y \text { is } B^{j} .
\end{aligned}
$$

This means that $A_{i}^{j}, i=1,2, \ldots, n$, are the fuzzy sets chosen from $A_{i l}$, where $l=1,2, \ldots, N_{i}$, which satisfies Eqn. (1), while $B^{j}$ equals the fuzzy set from $B_{l}$, where $l=1,2, \ldots, N_{y}$, which satisfies Eqn. (2). 
In the third step, a degree is assigned to each of the $M$ rules, with the use of (11) and (2), as follows:

$$
\begin{aligned}
& D\left(R^{j}\right) \\
& \quad=\mu_{A_{1}^{j}}\left(\bar{x}_{1}^{(j)}\right) \mu_{A_{2}^{j}}\left(\bar{x}_{2}^{(j)}\right) \ldots \mu_{A_{n}^{j}}\left(\bar{x}_{n}^{(j)}\right) \mu_{B^{j}}\left(\bar{y}^{(j)}\right) .
\end{aligned}
$$

As a matter of fact, the degree is obtained calculating the value of the rule firing level for the data pair that generated this rule, multiplied by the membership of the consequent fuzzy set.

The antecedent matching degree, also called the degree of activation of the rule $R^{j}$ or the rule firing level, is expressed by

$$
\tau_{j}=\prod_{i=1}^{n} \mu_{A_{i}^{j}}\left(\bar{x}_{i}\right)
$$

for $j=1,2, \ldots, M$.

Equations (4) and (5) can also be expressed in a more general form, by using a $T$-norm:

$$
\begin{aligned}
D\left(R^{j}\right)= & T\left\{\mu_{A_{1}^{j}}\left(\bar{x}_{1}^{(j)}\right), \mu_{A_{2}^{j}}\left(\bar{x}_{2}^{(j)}\right),\right. \\
& \left.\ldots, \mu_{A_{n}^{j}}\left(\bar{x}_{n}^{(j)}\right), \mu_{B^{j}}\left(\bar{y}^{(j)}\right)\right\}
\end{aligned}
$$

and

$$
\tau_{j}=T\left\{\mu_{A_{1}^{j}}\left(\bar{x}_{1}^{(j)}\right), \mu_{A_{2}^{j}}\left(\bar{x}_{2}^{(j)}\right), \ldots, \mu_{A_{n}^{j}}\left(\bar{x}_{n}^{(j)}\right)\right\} .
$$

The product, and the min operation are the most often used examples of $t$-norm functions.

The degree, $D\left(R^{j}\right)$, assigned to each rule $R^{j}, j=$ $1,2, \ldots, M$, allows reducing the number of the rules, including into the rule base of a fuzzy system only the rules with the maximal degree in particular regions. In this way, the problem of conflicting rules, i.e., those that have the same IF part but different THEN parts, is solved, resulting in the reduction of the initial number of rules.

Thus, starting from $M$ rules generated by $M$ data pairs, the Wang-Mendel algorithm produces the rule base of a fuzzy system composed of the reduced number of $N$ rules, where $N \leq M$.

The Mamdani type of a fuzzy system with inference based on $N$ fuzzy IF-THEN rules, generated by the Wang-Mendel method, can be described by the following mathematical model:

$$
\bar{y}=\frac{\sum_{k=1}^{N} \bar{y}^{k} \tau_{k}}{\sum_{k=1}^{N} \tau_{k}},
$$

where $\bar{y}^{k}, k=1,2, \ldots, N$, is is the point in which the membership function $\mu_{B^{k}}(y)$ takes the maximal value, and $\tau_{k}$ is given by (5), where $j$ is replaced by $k$ and $M$ by $N$.

It should be noted that $\tau_{1}, \tau_{2}, \ldots, \tau_{N}$ in Eqn. (8) are special cases of those determined using (7).
Introducing to the antecedents of the rules (8) their importance weights, we get

$$
\bar{y}=\frac{\sum_{k=1}^{N} w_{k} \bar{y}^{k} \tau_{k}}{\sum_{k=1}^{N} w_{k} \tau_{k}}
$$

which can describe the fuzzy recommender studied in this paper.

Remark 1. Although the formula (9) has been applied in the literature in various problems of classification and modeling (see, e.g., Alvarez-Estevez and Moret-Bonillo, 2018; Ishibuchi and Nakashima, 2001; Ishibuchi and Yamamoto, 2005; Nauck and Kruse, 1998; Rutkowski, 2004), for the first time it will be used in the context of designing fuzzy explainable recommenders. Moreover, our approach allows significantly reducing the number of rules.

Despite the fact that the usefulness of rule weighs is discussed in the literature (see, e.g., Simiński, 2010; Nauck and Kruse, 1998), we find this issue interesting from the interpretability and explainability point of view with regard to recommender systems. In general, the weights can be interpreted as the rule importance in the sense of expressing the number of data items in a dataset described by this rule. The more data items match the rule, the more important it is.

\section{Description of the proposed method}

This section presents the main algorithm proposed for the design of explainable recommender systems, including the procedure of rule reduction. The idea of the method is described in Section 3.1. Then, in Section 3.2, the procedure of feature encoding, introduced in order to apply the recommender to the MovieLens data, is presented in detail. In Section 3.3 three criteria are used for evaluating an optimal balance between the recommender's accuracy and interpretability. Finally, a short summary is included in Section 3.4

3.1. Idea of the proposed method. There are various methods of improving the performance of fuzzy systems, from introducing weights of the fuzzy rules (see, e.g., Ishibuchi and Nakashima, 2001), through reducing the number of the fuzzy rules (see, e.g., Cpalka, 2017), to optimizing a fuzzy system, usually by modifying fuzzy set parameters (see, e.g., Jin, 2000) or rule consolidation (see, e.g., Riid and Preden, 2017) and using the collaborative fuzzy clustering (see, e.g., Prasad et al., 2017).

In this paper, a hybrid solution is proposed in which system optimization is applied after successive reductions of fuzzy rules (see Algorithm 1). The optimization of the system, in this case, refers to optimized values of the weights. The rule reduction procedure should increase the accuracy of the system. 
In order to reduce the rules, the method of removing successively the least beneficial fuzzy rules was used. To find the least beneficial fuzzy rule, particular rules are turned off and on in the system one by one, and the system error is calculated after each change. The least beneficial rule is the one whose RMSE was the lowest after reduction.

As explained above, during the optimization of the system, we assume that only the weights (initialized equally by default) of the rules can be modified. For this purpose, we use the standard evolutionary strategy ES $(\mu+\lambda)$ (see, e.g., Rutkowski, 2008). However, any optimization algorithm can be applied instead. This approach allows keeping a semantically interpretable form of fuzzy sets and increasing system accuracy.

Moreover, this approach is more legible than the interpretation of systems in which fuzzy sets become uninterpretable after the optimization of their parameters (see, e.g., Jin, 2000). The optimized system will be labeled as WO in the remainder of this paper.

Two approaches will be considered: $\mathrm{C} 1$, in which values of the weights are reset each time the fuzzy rule is removed, and $\mathrm{C} 2$, in which the values of the weights are stored after the optimization and do not reset.

The performance of the system in which the values of the weights were rounded (to one decimal place) after the optimization was also tested. The goal was to increase the transparency of the fuzzy rule notation. The approach with the rounded weight values is marked in this paper as WR, while WO denotes the system with optimized weights (not rounded values).

The idea of the presented approaches is shown in Algorithm 1. System optimization and evaluation are repeated after the reduction of each fuzzy rule, with the goal of finding the best reduction level for which the weight values give the best system performance. The evaluation is illustrated in Section 4.

It is worth adding that, as part of the tests, the results for a given number of rules and a specific reduction level were saved and then averaged. This is due to the fact that, in recommendation systems, the datasets for each user are different so a different number of fuzzy rules is created by using the WM (Wang-Mendel) method.

3.2. Feature encoding. Objects (items) that can be recommended are described by attributes (features). In recommender systems, attribute values can be of different type, not necessarily crisp (numerical). In general, an object is characterized by attributes whose values can be numerical or categorical (nominal), and other data types can be considered as well.

Denote by $\mathbf{a}=\left[a_{1}, a_{2}, \ldots, a_{n}\right]^{T}$ a vector of attributes describing an object $\mathbf{o}_{j}$ that belongs to $\mathbf{O}$ that is a space of the objects. The vector a corresponds to

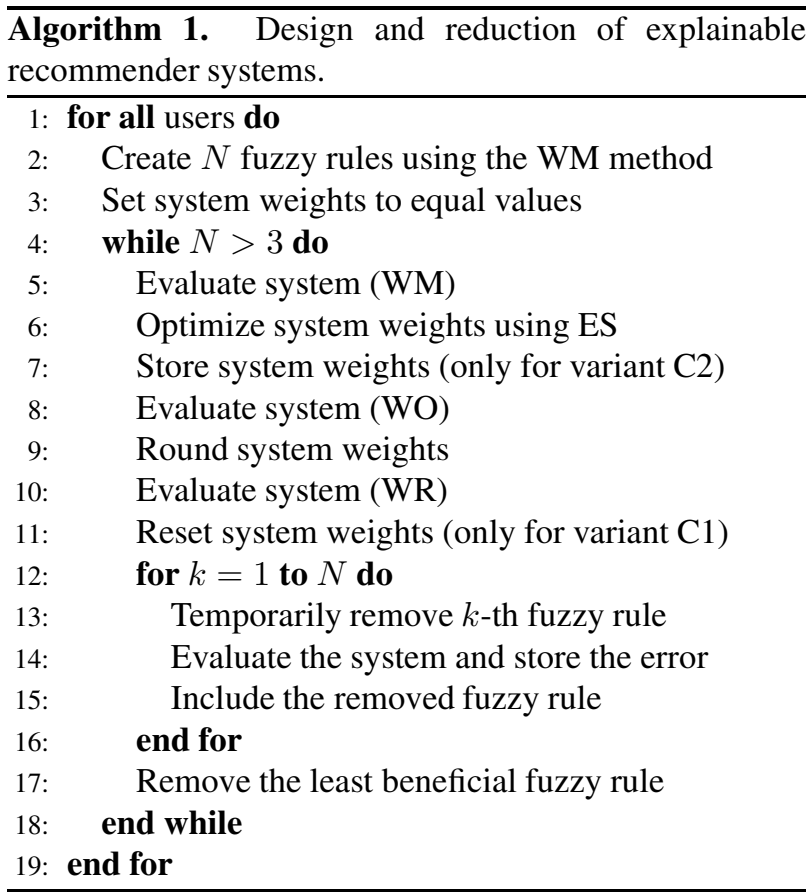

the vector of linguistic variables $\mathbf{x}=\left[x_{1}, x_{2}, \ldots, x_{n}\right]^{T}$, considered in Section 2 .

Now, let us focus our attention on attribute values. In the case of categorical (nominal, linguistic) values, we can use symbols $N_{i}$, for $i=1,2, \ldots, n$, and $N_{y}$ concerning both the number of values of attributes (input variables) and output (decision) variables, respectively. Fuzzy sets as linguistic values can be viewed as special cases of categorical values.

Let $\mathbf{v}=\left[v_{1}, v_{2}, \ldots, v_{n}\right]^{T}$ denote a vector of values of particular attributes $a_{i}$, for $i=1,2, \ldots, n$, in general, and let $\mathbf{v}^{j}=\left[v_{1}{ }^{j}, v_{2}{ }^{j}, \ldots, v_{n}{ }^{j}\right]^{T}$ represent this vector for the object $\mathbf{o}_{j}$, where $j=1,2, \ldots, M$. It is worth noticing that the vector $\mathbf{v}^{j}$ corresponds to the vector $\overline{\mathbf{x}}^{(j)}=\left[\bar{x}_{1}{ }^{(j)}, \bar{x}_{2}{ }^{(j)}, \ldots, \bar{x}_{n}{ }^{(j)}\right]^{T}$, considered in Section2

However, with regard to recommender systems, objects may be characterized by attributes that take more than only one value, and not necessarily values belonging to the set of reals.

For example, when movies are considered, their attributes such as genre can be described by categorical values, e.g., action, comedy, drama, and there are movies characterized, e.g., as comedy and drama.

Let $V_{i}=\left\{V_{i 1}, V_{i 2}, \ldots, V_{i N_{i}}\right\}$ be a set of values of the attribute $a_{i}$, for $i=1,2, \ldots, n$. The notation of the attribute values corresponds to fuzzy linguistic values, $A_{i l}$, for $i=1,2, \ldots, n$ and $l=1,2, \ldots, N_{i}$, presented in Section 2

For the above-mentioned example, $a_{i}$ can be genre and $V_{i}=\{$ action, comedy,drama,... $\}$. Of course, each object $\mathbf{o}_{j}, j=1,2, \ldots, M$, which is a movie in this case, is characterized by attributes with values taken from 
the same set of the attribute values.

Let $d_{j}, j=1,2, \ldots, M$, be the score (rating) of the object $\mathbf{o}_{j}$, given by a user. With regard to the movies, users assess chosen movies by using grades from the set $\{2,3,4,5\}$ of the user's preference values that express how much the user likes a movie.

The rating values, in a movie dataset (e.g., MovieLens), correspond to the output values $\bar{y}^{(j)}$ in the input-output data pairs, considered in Section 2, with regard to the output variable $y \in Y$. The number of output values, $N_{y}$, in the case of discrete values of the grades $\{2,3,4,5\}$ equals 4 .

Let us define the set of matrices $\mathbf{C}^{(i)}=\left[c_{j l}{ }^{(i)}\right]_{M \times N_{i}}$, for $i=1,2, \ldots, n$, where $c_{j l}{ }^{(i)}$, for $j=1,2, \ldots, M$ and $l=1,2, \ldots, N_{i}$, are determined according to the following equation:

$$
c_{j l}{ }^{(i)}= \begin{cases}d_{j} & \text { if } V_{i l} \text { is in object } \mathbf{o}_{j} \\ 0 & \text { otherwise }\end{cases}
$$

It should be explained that each matrix $\mathbf{C}^{(i)}$, $i=1,2, \ldots, n$, includes $M$ rows corresponding to $M$ objects (items), e.g., movies, and $N_{i}$ columns labeled by the values $V_{i 1}, V_{i 2}, \ldots, V_{i N_{i}}$ of the attribute $a_{i}$. The elements $c_{j l}{ }^{(i)}$ of the matrix $\mathbf{C}^{(i)}$, for $j=1,2, \ldots, M$ and $l=1,2, \ldots, N_{i}$, equal $d_{j}$ if the attribute value $V_{i l}$ occurs in an object $\mathbf{o}_{j}$, and zero otherwise. With regard to the example of the movie characterized as comedy and drama, every element in the row assigned to this movie equals zero except for those referring to comedy and drama.

In order to apply the fuzzy system described in Section 2 as the recommender, we need $M$ input-output data pairs $\overline{\mathbf{x}}^{(j)}, \bar{y}^{(j)}, j=1,2, \ldots, M$, and $\overline{\mathbf{x}}^{(j)}=\left[\bar{x}_{1}{ }^{(j)}, \bar{x}_{2}{ }^{(j)}, \ldots, \bar{x}_{n}{ }^{(j)}\right]^{T}$. As mentioned earlier, this corresponds to the pairs $\mathbf{v}^{j}, d_{j}$, where $\mathbf{v}^{j}=\left[v_{1}{ }^{j}, v_{2}{ }^{j}, \ldots, v_{n}{ }^{j}\right]^{T}$ represents single numerical values referring to particular attributes $a_{i}^{j}, i=1,2, \ldots, n$, of an object $\mathbf{o}_{j}, j=1,2, \ldots, M$.

We call the single values that characterize the attributes the attribute preferences, and determine them based on the attribute value preferences in the following way:

$$
\psi\left(V_{i l}\right)=\frac{\sum_{j=1}^{M} c_{j l}^{(i)}}{m_{l}^{(i)}},
$$

where $\psi$ means the preference and $m_{l}{ }^{(i)}$ denotes the number of non-zero elements in the $l$-th column of the matrix $\mathbf{C}^{(i)}, i=1,2, \ldots, n$.

Then, the attribute preference, $v_{i}{ }^{j}$, is determined as

$$
v_{i}^{j}=\frac{\sum_{l=1}^{\alpha_{j}{ }^{(i)}} \psi^{*}\left(V_{i l}\right)}{\alpha_{j}{ }^{(i)}},
$$

Examples from the database, for a user:

movie 1 - genre $\{$ action, comedy $\}, \ldots$, user rate $=5.0$ movie 2 - genre $\{$ drama, comedy $\}, \ldots$, user rate $=4.0$ movie 3 - genre $\{$ drama $\}, \ldots$, user rate $=2.0$

movie 4 - genre $\{$ action, drama $\}, \ldots$, user rate $=3.0$

Preference of attribute values for genre:

action - preference $=(5.0+3.0) / 2=4.0$

comedy - preference $=(5.0+4.0) / 2=4.5$

drama - preference $=(4.0+2.0+3.0) / 3=3.0$

Dataset prepared for the fuzzy system:

movie 1 - genre preference $\{4.25\}, \ldots$, user rate $=5.0$ movie 2 - genre preference $\{3.75\}, \ldots$, user rate $=4.0$ movie 3 - genre preference $\{3.00\}, \ldots$, user rate $=2.0$ movie 4 - genre preference $\{3.50\}, \ldots$, user rate $=3.0$

Fig. 1. Example of preparation of the dataset for a user.

for $i=1,2, \ldots, n$ and $j=1,2, \ldots, M$, where $\psi^{*}$ denotes the preference of only those attribute values $V_{i l}$, $l=1,2, \ldots, N_{i}$, that are included as values of the object $\mathbf{o}_{j}$, and $\alpha_{j}{ }^{(i)}$ is the number of $\psi^{*}\left(V_{i l}\right)$.

Figure 1 illustrates the above-described process of determining the attribute value preferences, according to Eqn. (11), and the results of the attribute preference obtained by use of the formula (12). This simple example refers to $M=4$ movies as the objects $\mathbf{o}_{j}, j=1,2,3,4$, assessed by a user with the ratings 5, 4, 2, and 3, respectively. This means that $d_{1}=5, d_{2}=4, d_{3}=$ $2, d_{4}=3$. The attribute genre is considered, and $\mathbf{0}_{1}$ is characterized by two attribute values, i.e., action and comedy, object $\mathbf{o}_{2}$ by drama and comedy, object $\mathbf{0}_{3}$ by only one value, i.e., drama, and $\mathbf{o}_{4}$ by action and drama. Therefore, the attribute value preferences, $\psi$, are calculated for action, comedy, and drama as $V_{i l}$, for $l=1,2,3$ and $i$ referring to the genre as the attribute.

It is very easy to present matrix $\mathbf{C}^{(i)}$ for this simple example. The following genre preference values were obtained in this case: 4.25 for $\mathbf{o}_{1}, 3.75$ for $\mathbf{0}_{2}, 3.00$ for $\mathbf{o}_{3}$, and 3.50 for $\mathbf{o}_{4}$. In the same way, we calculate the preference values for other attributes, and all of them are included in the data pairs $\mathbf{v}^{j}, d_{j}$, for the fuzzy recommender. It should be emphasized that this example concerns only one user with regard to the recommendation system. The same procedure is repeated for other users.

3.3. Solution evaluation. Since the rates of objects are numerical values, the rules obtained by the WM method are used in this paper as the base for the Mamdani type fuzzy system for regression tasks (see, e.g., Cpalka, 2017 ) with the goal of user rate prediction. This allows calculating the RMSE (commonly used error definition, 
known as the root mean square error), and also obtaining more accurate classification of recommended objects.

In addition to the RMSE, the accuracy (ACC) of predictions of the exact user rate were calculated. To make this possible, the system's output values were rounded to the possible values of the object's rate and in this way the standard classification error (ACC) could be calculated (see, e.g., Kuncheva, 2000). There was also a classification error (YES/NO), checking whether or not an item (object) should be recommended (if the value of its recommendation is more than a half of possible values). This approach is considered in some other papers.

Because the proposed method allows obtaining a different system accuracy for a different reduction level of fuzzy rules, choosing the optimal balance in terms of accuracy-interpretability is not a trivial task. In this paper, the use of isocriterial lines and criteria for model evaluation with regard to their complexity is proposed (see, e.g., Söderström and Stoica, 1989).

The first criterion considered is the Akaike information criterion (AIC), which is defined as follows:

$$
\mathrm{AIC}=M \cdot \ln Q+2 \cdot p,
$$

where $M$ stands for the number of dataset samples (in this paper this value was set to the average number of the dataset samples generated for all users), $Q$ denotes the system error, $p$ means parameters that are optimized in the system (equal to the number of weights and analogously the number of fuzzy rules).

The second criterion used is the final prediction error (FPE), defined as follows:

$$
\mathrm{FPE}=Q \cdot\left(\frac{M \cdot n+p}{M \cdot n-p}\right),
$$

where $n$ denotes the number of system inputs.

The last criterion considered is the Schwarz criterion which is expressed by the following equation:

$$
S=M \cdot \ln Q+p \cdot \ln M .
$$

The isocriterial lines are lines representing fixed values of the criteria with different values of system errors and the number of system parameters. This approach allows solving the problem of a compromise between the system error and the number of optimized parameters describing the system. The points located on the isocriterial lines, with the smallest criterion values, characterize systems that are called sub-optimal. These provide the smallest values of statistical criteria within the examined structures (in this case, differing in the number of system rules).

3.4. Summary of the proposed method. The proposed approach (a) is based on the WM method, which allows generating interpretable and simple fuzzy rules, (b) uses weights' optimization and does not modify fuzzy sets parameters in order to keep interpretable fuzzy sets, (c) applies fuzzy rule reduction not only to simplify the system but also to decrease the system error, (d) allows checking how the optimization of system weights works with a different level of rule reduction, (e) employs the standard ES for weight optimization, (f) includes a version that rounds fuzzy rules weights to increase the system's interpretability, and (g) uses isocriterial lines in order to keep the optimal balance between the system interpretability and system error.

\section{System performance evaluation}

Simulations that illustrate the performance of the system proposed for recommendation are described in this section. The results of the simulations verify the effectiveness of the recommender system for the MovieLens 10M dataset. The datasets are available on the Internet as versions of different sizes (see Harper and Konstan, 2015). These datasets contain user ratings for movies.

4.1. Description of computer simulations. In the simulations, the cases described in Section 3.1 and presented in Table 1 were tested. In case $\mathrm{C} 1$, the weight values are reset after each reduction of a fuzzy rule, while in case $\mathrm{C} 2$, weight, the values are stored after optimization and do not reset (see Algorithm 1).

Table 1. Simulation cases

\begin{tabular}{|c|c|c|}
\hline Case & Variant & Weight values \\
\hline \hline WM & - & not optimized \\
WO-C1 & C1 & optimized and reset \\
WR-C1 & C1 & optimized, rounded, and reset \\
WO-C2 & C2 & optimized and do not reset \\
WR-C2 & C2 & optimized, rounded, do not reset \\
\hline
\end{tabular}

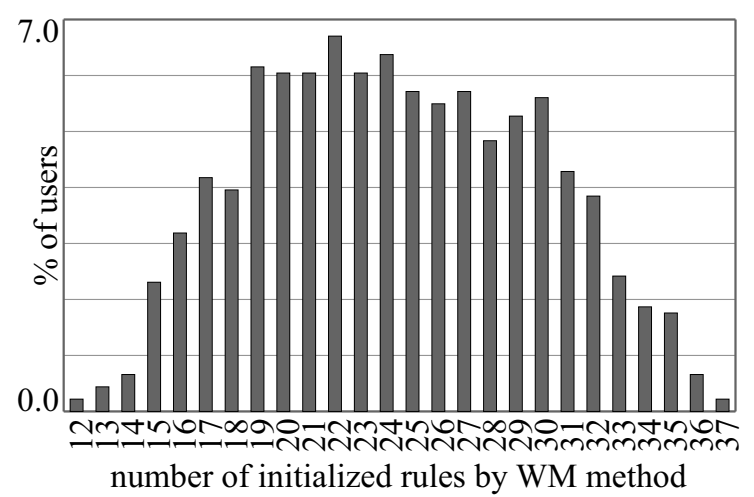

Fig. 2. Histogram of the initial number of rules generated with the use of the WM method. 
The number of fuzzy sets (linguistic values), $N_{i}$, for each attribute corresponding to linguistic variables $x_{i}, i=$ $1,2, \ldots, n$, was chosen as Gaussian-type fuzzy sets, for $N_{i}=5$. The following parameters of the ES algorithm were set: population size $=32$, number of iterations $=$ 100 , evaluation function $=$ RMSE.

For the simulations, the MovieLens 10M database is used, and three inputs, $n=3$, are considered: genre preference (explained in Section 3.2), year (numeric values), and keywords preference. Moreover, datasets were prepared for the first 100 users who rated more than 30 movies from the database.

As a testing method, 10-fold cross-validation was applied, and only learning dataset samples were taken for creating fuzzy rules with the WM method (the remaining dataset samples are used as testing samples).

Since rating predictions for unknown samples is important in recommendation systems, the error values for testing samples are crucial while comparing the results. Therefore, most of the conclusions in this paper are focused on this analysis (see Tables 24.

4.2. Simulation results. The histogram of the initial number of rules generated by using the WM method is shown in Fig. 2. Depending on a user and the number of rated objects, the WM generates different numbers of fuzzy rules, with an average of 24 rules for the proposed feature encoding. The number of rules for particular users differs from 12 to 37 .

Detailed simulation results are presented in Tables 2 (RMSE), 3 (ACC) and 4 (YES/NO). The values of the criteria are included in Tables 5 and 6 . The isolines, for the AIC, FPE and Schwarz criteria, are illustrated in Figs. 4 and 5

A comparison of the WM, WO-C1, WR-C1, WO-C2 and WR-C2 cases is shown in Figs. 6, 7 and 8, The optimization process of weights for different numbers of fuzzy rules is presented in Fig. 9, and the histogram of accuracy obtained for different users is portrayed in Fig. 3. In addition, examples of fuzzy sets and fuzzy rules obtained for the presented methods are shown in Tables 7 (according to the reduction level of fuzzy rules considering obtained accuracy) and 8 (according to the reduction in fuzzy rules for the AIC, FPE and Schwarz criteria).

Analyzing Tables 2 and 3, we see that $32 \%-36 \%$ of the rules generated by the WM method can be removed, resulting in better performance of the system on both learning and testing data samples, with regard to the average RMSE and ACC, although in Table 4, where the YES/NO classification error is considered, the same result is obtained only for the learning samples but not for the testing samples. It is worth noticing that the value of the YES/NO error in the case of $36 \%$ rule reduction does not differ much from the value of this error for the case of $0 \%$, i.e., no rule reduction. This means that $36 \%$ of the rules can be removed, but not more in all the cases of the WM system.

Similar results were obtained for the WO-C2 system, which is the best case compared with WO-C1, WR-C1,
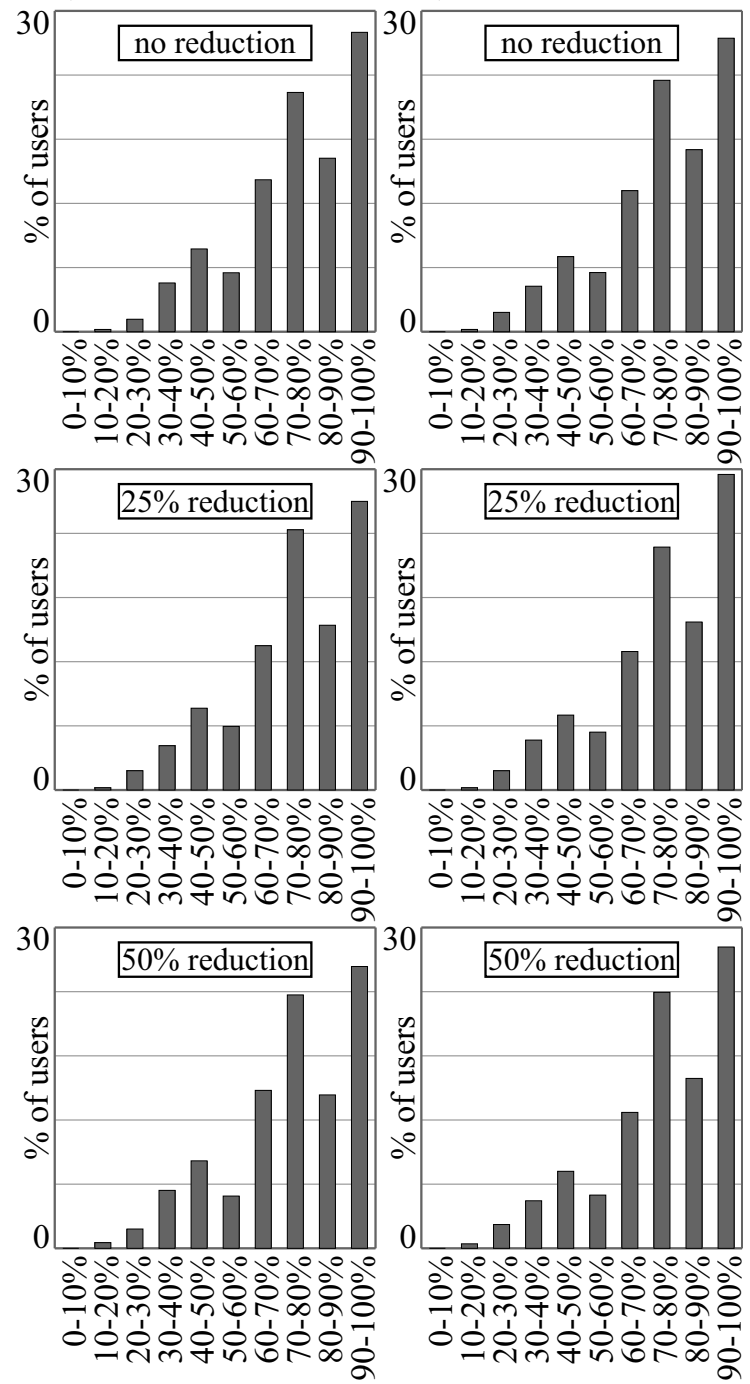

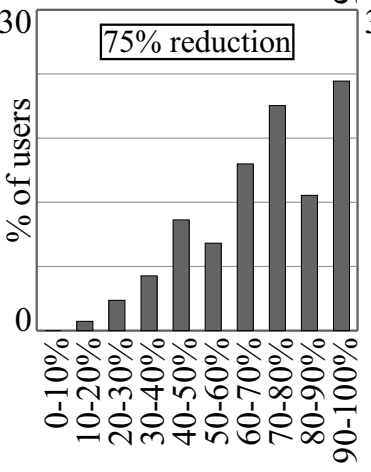

(a)WO-C1

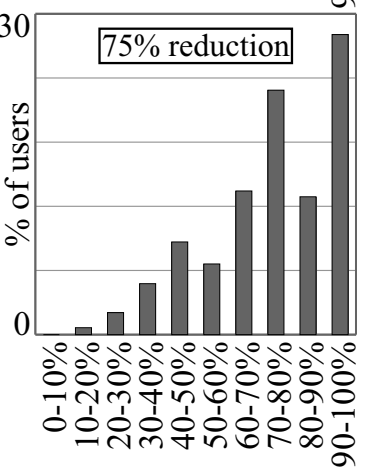

(b)WO-C2
Fig. 3. Histogram of accuracy (ACC) obtained for different users. 


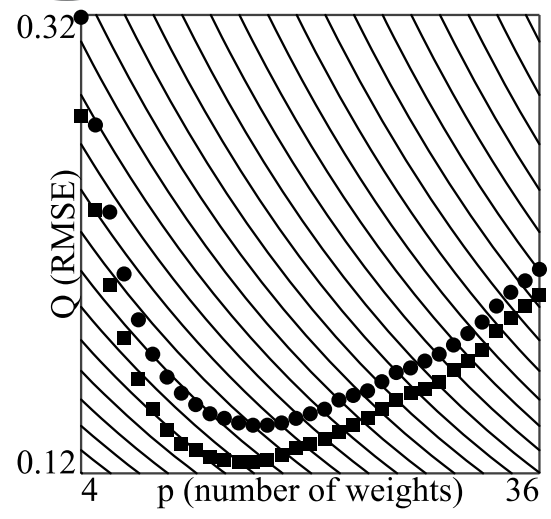

(a)Akaike (AIC)

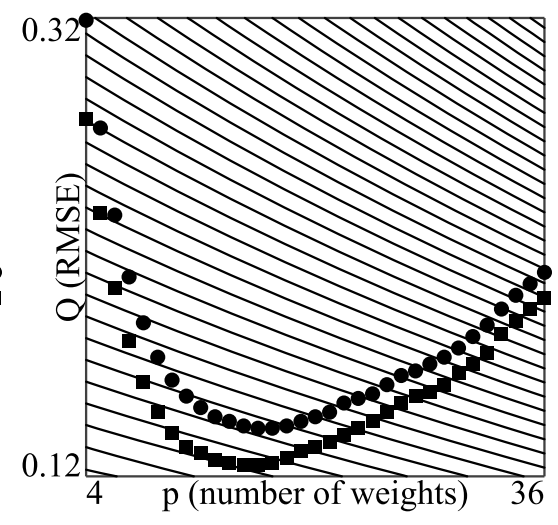

(b) FPE

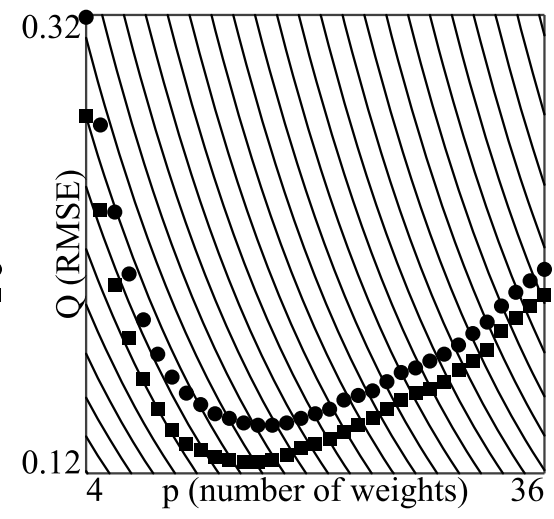

(c) Schwarz (S)

Fig. 4. Isolines lines for the learning samples, where circles and squares represent the WO-C1 and WO-C2 systems, respectively.

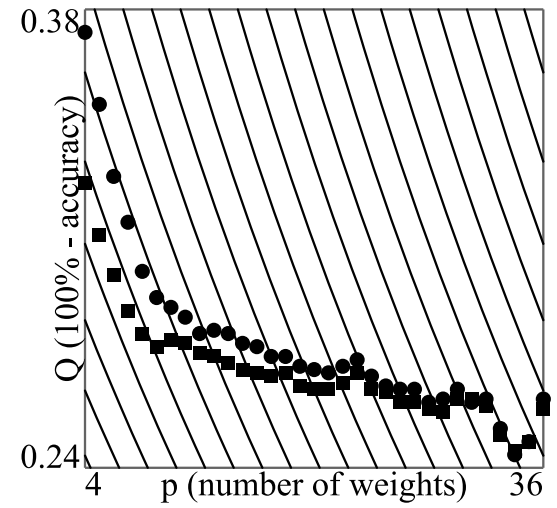

(a) Akaike (AIC)

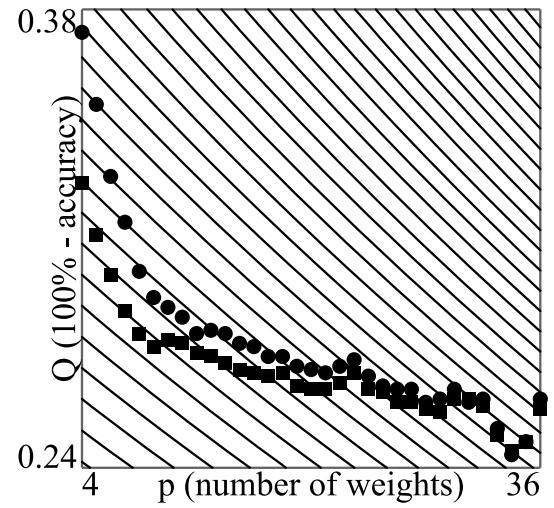

(b) FPE

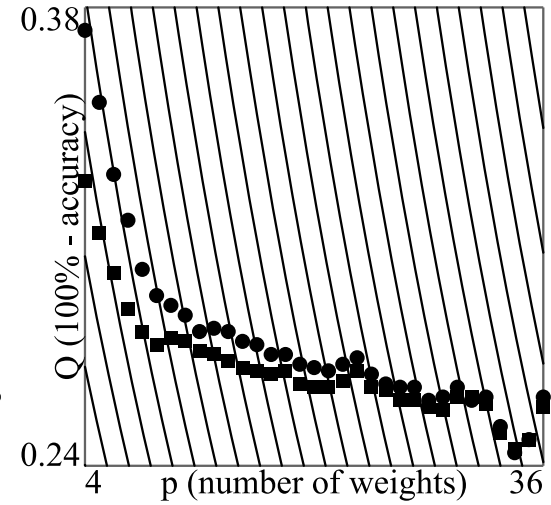

(c) Schwarz (S)

Fig. 5. Isolines lines for the testing samples, where circles and squares represent the WO-C1 and WO-C2 systems, respectively.

and WR-C2. Figures 4 and 5 as well as Figs. 6, 7 and 8. show the best performance of the WO-C2 system. In addition, Fig. 3 illustrates better accuracy of the WO-C2 system than WO-C1, and the best for $25 \%$ rule reduction (or probably more) but less than $50 \%$.

Figure 9 also portrays better performance of the WO-C2 system than that of WO-C1. However, with regard to the RMSE, it shows that about 20 rules give a good result for both learning and testing data samples but more (30 rules) are worse for the learning samples, but best for the testing data samples. It seems obvious that it is possible to obtain better results by reducing the number of rules for learning data, yet the reduced number of rules may not always be sufficient for testing data.

Looking at Fig. 4] and Table 5, we see that the value of parameter $p$, which corresponds to the number of weights (and hence the number of fuzzy rules), equals 11 for the AIC, 14 for the FPE, and 9 for the Schwarz criterion. Thus, these criteria indicate the number of rules that can be removed with an optimal balance in terms of system accuracy and interpretability.
The obtained fuzzy rules are semantically interpretable. For example, rule number 5 from Table 77. "IF genre pref. is high and year is medium and keywords pref. is high THEN user rate is high" can be used as an explanation, as the antecedents of the rule are direct reasons for the recommendation. With this approach, it is also possible to refer the user to the visualization of the particular fuzzy set describing the meaning of the following terms: "high genre pref.", "medium year" and "high keywords pref.". The number of rules is low, and sufficient for good accuracy of the recommender system. Moreover, such a rule base allows explaining the performance of the system with regard to the recommendations (see Tables 7 and 8 , with Figs. 10 and 11, which illustrate the fuzzy rules for two users).

\section{Conclusions and final remarks}

The most important issue in this paper is the explainable fuzzy recommender. The explainability is realized by using semantically interpretable fuzzy IF-THEN rules. Examples of such rules are presented in Tables 7 and 8 
Table 2. Average RMSE for all users in terms of the percentage of reduced rules (RR).

\begin{tabular}{|c|c|c|c|c|c|c|c|c|c|c|}
\hline \multirow{2}{*}{$\begin{array}{l}\% \text { of } \\
\text { RR }\end{array}$} & \multicolumn{5}{|c|}{ learning samples } & \multicolumn{5}{|c|}{ testing samples } \\
\hline & $\overline{\mathrm{WM}}$ & WO-C1 & WR-C1 & WO-C2 & WR-C2 & $\overline{\mathrm{WM}}$ & WO-C1 & WR-C1 & $\mathrm{WO}-\mathrm{C} 2$ & WR-C2 \\
\hline $0 \%$ & 0.2199 & 0.1425 & 0.1485 & 0.1423 & 0.1472 & 0.3096 & 0.2670 & 0.2688 & 0.2677 & 0.2701 \\
\hline $4 \%$ & 0.2302 & 0.1605 & 0.1637 & 0.1513 & 0.1580 & 0.3023 & 0.2641 & 0.2669 & 0.2619 & 0.2648 \\
\hline $8 \%$ & 0.2106 & 0.1518 & 0.1582 & 0.1408 & 0.1560 & 0.2980 & 0.2674 & 0.2698 & 0.2658 & 0.2719 \\
\hline $12 \%$ & 0.1915 & 0.1395 & 0.1470 & 0.1283 & 0.1465 & 0.2910 & 0.2670 & 0.2706 & 0.2635 & 0.2724 \\
\hline $16 \%$ & 0.1908 & 0.1448 & 0.1511 & 0.1316 & 0.1534 & 0.2890 & 0.2688 & 0.2713 & 0.2638 & 0.2734 \\
\hline $20 \%$ & 0.1876 & 0.1462 & 0.1535 & 0.1329 & 0.1595 & 0.2876 & 0.2693 & 0.2713 & 0.2662 & 0.2765 \\
\hline $24 \%$ & 0.1807 & 0.1421 & 0.1497 & 0.1282 & 0.1576 & 0.2858 & 0.2669 & 0.2701 & 0.2600 & 0.2731 \\
\hline $28 \%$ & 0.1801 & 0.1429 & 0.1527 & 0.1279 & 0.1638 & 0.2932 & 0.2755 & 0.2791 & 0.2682 & 0.2863 \\
\hline $32 \%$ & 0.1789 & 0.1438 & 0.1531 & 0.1290 & 0.1684 & 0.2914 & 0.2748 & 0.2777 & 0.2683 & 0.2906 \\
\hline $36 \%$ & 0.1775 & 0.1435 & 0.1540 & 0.1285 & 0.1716 & 0.2843 & 0.2698 & 0.2741 & 0.2637 & 0.2848 \\
\hline $40 \%$ & 0.1801 & 0.1463 & 0.1580 & 0.1299 & 0.1804 & 0.2896 & 0.2750 & 0.2795 & 0.2692 & 0.2937 \\
\hline $44 \%$ & 0.1803 & 0.1465 & 0.1583 & 0.1299 & 0.1794 & 0.2949 & 0.2779 & 0.2823 & 0.2713 & 0.2956 \\
\hline $48 \%$ & 0.1797 & 0.1455 & 0.1594 & 0.1278 & 0.1869 & 0.2917 & 0.2739 & 0.2782 & 0.2683 & 0.2958 \\
\hline $52 \%$ & 0.1892 & 0.1519 & 0.1663 & 0.1325 & 0.1910 & 0.2971 & 0.2776 & 0.2826 & 0.2721 & 0.2994 \\
\hline $56 \%$ & 0.1956 & 0.1561 & 0.1741 & 0.1353 & 0.2002 & 0.3026 & 0.2793 & 0.2858 & 0.2738 & 0.3039 \\
\hline $60 \%$ & 0.1993 & 0.1592 & 0.1830 & 0.1375 & 0.2120 & 0.3048 & 0.2845 & 0.2935 & 0.2747 & 0.3057 \\
\hline $64 \%$ & 0.2143 & 0.1708 & 0.1955 & 0.1467 & 0.2240 & 0.3209 & 0.2941 & 0.3042 & 0.2802 & 0.3110 \\
\hline $68 \%$ & 0.2292 & 0.1826 & 0.2139 & 0.1570 & 0.2429 & 0.3272 & 0.2968 & 0.3067 & 0.2806 & 0.3189 \\
\hline $72 \%$ & 0.2442 & 0.1963 & 0.2321 & 0.1691 & 0.2582 & 0.3291 & 0.2980 & 0.3127 & 0.2813 & 0.3280 \\
\hline $76 \%$ & 0.2691 & 0.2179 & 0.2585 & 0.1886 & 0.2902 & 0.3532 & 0.3192 & 0.3364 & 0.2932 & 0.3448 \\
\hline $80 \%$ & 0.3025 & 0.2453 & 0.2967 & 0.2168 & 0.3166 & 0.3715 & 0.3305 & 0.3532 & 0.2978 & 0.3538 \\
\hline $84 \%$ & 0.3450 & 0.2826 & 0.3513 & 0.2465 & 0.3504 & 0.3892 & 0.3415 & 0.3758 & 0.3024 & 0.3638 \\
\hline $88 \%$ & 0.3945 & 0.3289 & 0.3950 & 0.2815 & 0.3776 & 0.4229 & 0.3680 & 0.4089 & 0.3233 & 0.3830 \\
\hline $92 \%$ & 0.4406 & 0.3642 & 0.4229 & 0.3045 & 0.4021 & 0.4685 & 0.3943 & 0.4311 & 0.3344 & 0.4035 \\
\hline
\end{tabular}

Table 3. Average ACC for all users in terms of the percentage of reduced rules (RR).

\begin{tabular}{|c|c|c|c|c|c|c|c|c|c|c|}
\hline \multirow{2}{*}{$\begin{array}{c}\% \text { of } \\
\text { RR }\end{array}$} & \multicolumn{5}{|c|}{ learning samples } & \multicolumn{5}{|c|}{ testing samples } \\
\hline & WM & WO-C1 & WR-CI & WO-C2 & WR-C2 & WM & WO-C1 & WR-CI & WO-C2 & WR-C2 \\
\hline$\overline{0 \%}$ & 77.699 & 91.091 & 90.600 & 91.019 & $\overline{90.607}$ & 67.149 & $\overline{76.721}$ & $\overline{76.469}$ & 76.463 & 75.903 \\
\hline $4 \%$ & 76.523 & 89.049 & 88.595 & 90.213 & 89.533 & 67.095 & 75.701 & 75.783 & 76.087 & 75.969 \\
\hline $8 \%$ & 79.674 & 89.838 & 89.337 & 91.279 & 90.114 & 68.622 & 75.684 & 75.424 & 76.433 & 75.760 \\
\hline $12 \%$ & 82.977 & 91.313 & 90.892 & 92.679 & 91.370 & 70.778 & 76.154 & 75.830 & 76.810 & 75.908 \\
\hline $16 \%$ & 83.371 & 90.870 & 90.444 & 92.481 & 90.734 & 71.440 & 75.601 & 75.407 & 76.254 & 75.303 \\
\hline $20 \%$ & 83.562 & 90.565 & 90.018 & 92.221 & 90.126 & 71.341 & 75.424 & 75.047 & 76.408 & 75.142 \\
\hline $24 \%$ & 84.641 & 90.913 & 90.450 & 92.629 & 90.446 & 71.924 & 76.007 & 75.488 & 77.361 & 76.089 \\
\hline $28 \%$ & 85.113 & 91.018 & 90.458 & 92.871 & 90.215 & 72.212 & 75.628 & 75.451 & 76.992 & 75.490 \\
\hline $32 \%$ & 85.344 & 90.832 & 90.217 & 92.753 & 89.784 & 72.393 & 75.514 & 75.222 & 76.691 & 74.724 \\
\hline $36 \%$ & 85.340 & 90.644 & 89.970 & 92.654 & 89.499 & 72.715 & 75.504 & 75.011 & 76.682 & 74.880 \\
\hline $40 \%$ & 84.921 & 90.408 & 89.694 & 92.568 & 88.833 & 72.458 & 75.258 & 74.652 & 76.504 & 74.453 \\
\hline $44 \%$ & 84.882 & 90.415 & 89.615 & 92.602 & 88.959 & 71.478 & 74.953 & 74.275 & 76.147 & 73.934 \\
\hline $48 \%$ & 84.744 & 90.372 & 89.512 & 92.774 & 88.731 & 72.012 & 75.177 & 74.604 & 76.137 & 73.853 \\
\hline $52 \%$ & 83.361 & 89.594 & 88.683 & 92.266 & 88.184 & 71.875 & 75.049 & 74.671 & 76.568 & 74.182 \\
\hline $56 \%$ & 82.466 & 89.009 & 87.846 & 91.903 & 87.524 & 70.927 & 75.035 & 74.588 & 76.575 & 74.082 \\
\hline $60 \%$ & 81.833 & 88.428 & 87.055 & 91.445 & 86.700 & 70.369 & 74.356 & 73.855 & 76.099 & 73.787 \\
\hline $64 \%$ & 80.098 & 87.106 & 85.554 & 90.440 & 85.515 & 68.854 & 73.377 & 72.607 & 75.521 & 72.940 \\
\hline $68 \%$ & 78.280 & 86.052 & 84.154 & 89.407 & 83.852 & 68.518 & 73.879 & 73.221 & 75.819 & 73.069 \\
\hline $72 \%$ & 76.838 & 84.565 & 82.632 & 88.170 & 82.539 & 68.159 & 73.075 & 72.258 & 75.639 & 72.642 \\
\hline $76 \%$ & 74.454 & 82.450 & 80.187 & 86.261 & 80.290 & 65.625 & 71.480 & 70.541 & 74.488 & 71.682 \\
\hline $80 \%$ & 71.005 & 79.897 & 77.270 & 83.562 & 78.153 & 64.340 & 70.986 & 69.363 & 74.530 & 71.608 \\
\hline $84 \%$ & 67.335 & 75.982 & 72.789 & 80.602 & 74.940 & 62.052 & 68.947 & 67.010 & 73.520 & 70.176 \\
\hline $88 \%$ & 62.994 & 71.200 & 68.071 & 77.023 & 71.904 & 58.543 & 66.360 & 64.570 & 71.274 & 67.985 \\
\hline $92 \%$ & 59.315 & 68.213 & 65.502 & 74.907 & 70.161 & 54.622 & 64.517 & 62.078 & 70.704 & 66.941 \\
\hline
\end{tabular}



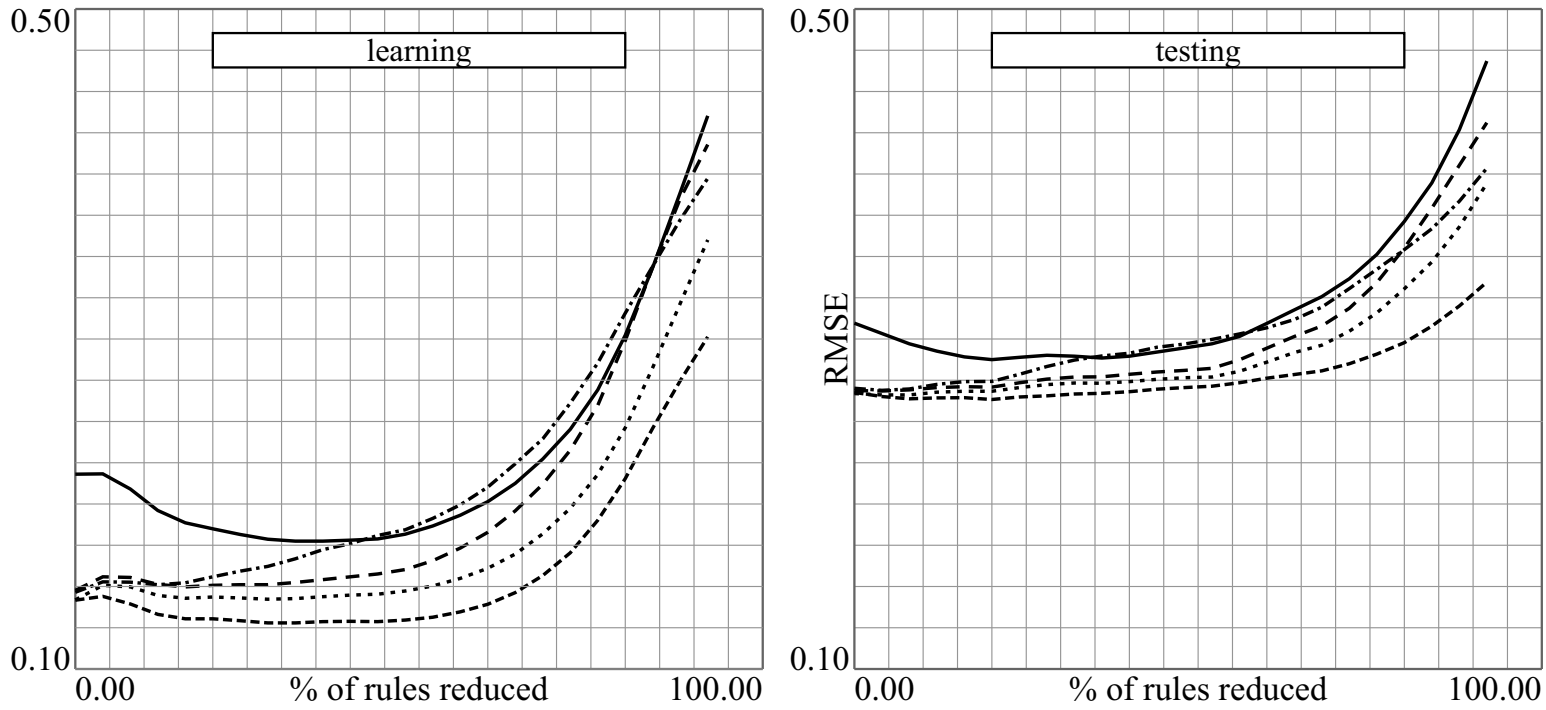

$\mathrm{WM}$

WO-C1

WR-C1

WO-C2

WR-C2

Fig. 6. Comparison of simulation cases in terms of the RMSE and the percentage of reduced rules.
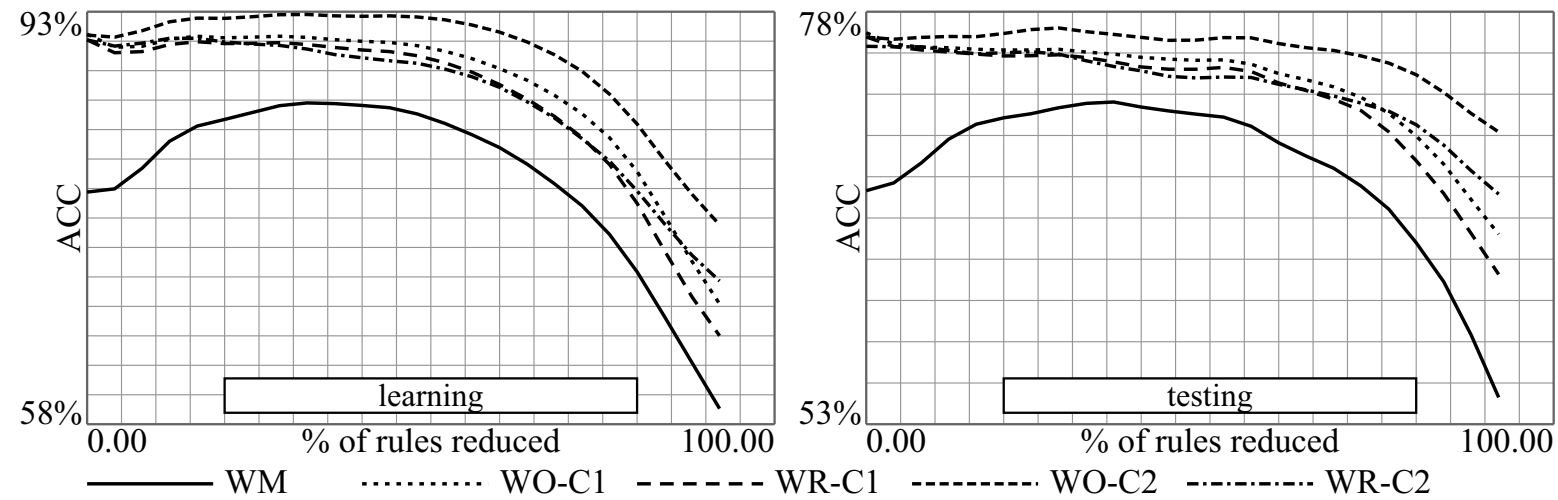

Fig. 7. Comparison of simulation cases in terms of ACC and the percentage of reduced rules.
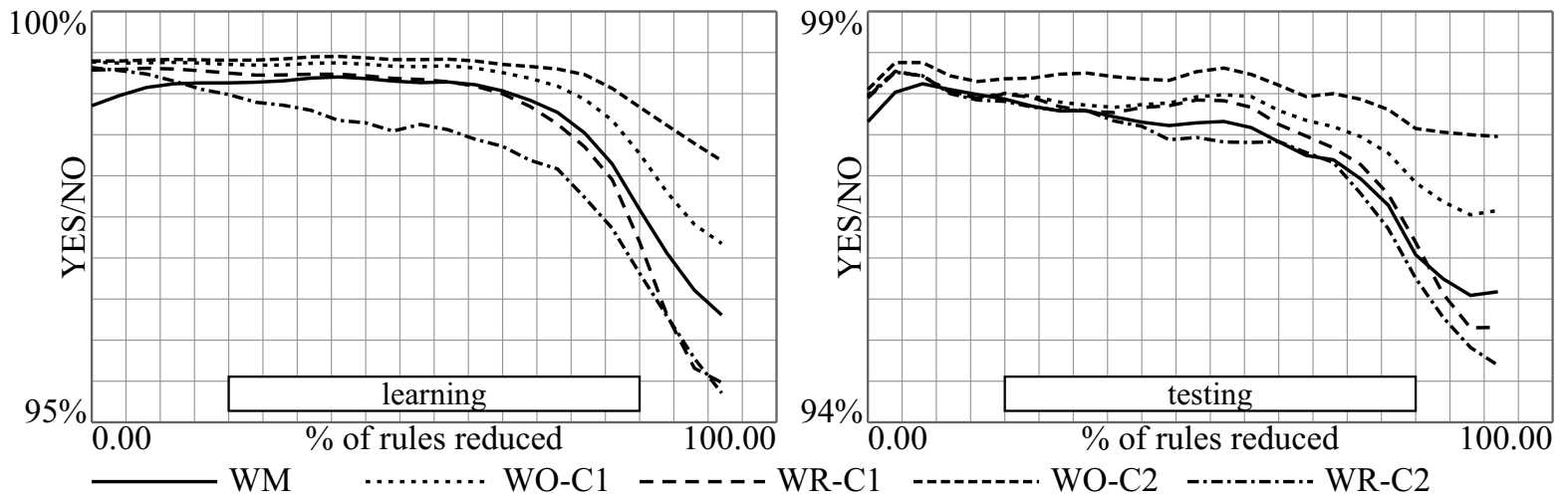

Fig. 8. Comparison of simulation cases in terms of YES/NO and the percentage of reduced rules. 
Table 4. Average YES/NO for all users in terms of the percentage of reduced rules (RR).

\begin{tabular}{|c|c|c|c|c|c|c|c|c|c|c|}
\hline \multirow{2}{*}{$\begin{array}{l}\% \text { of } \\
\text { RR }\end{array}$} & \multicolumn{5}{|c|}{ learning samples } & \multicolumn{5}{|c|}{ testing samples } \\
\hline & $\overline{\mathrm{WM}}$ & WO-C1 & WR-C1 & WO-C2 & WR-C2 & $\overline{\mathrm{WM}}$ & WO-C1 & WR-C1 & WO-C2 & WR-C2 \\
\hline$\overline{0 \%}$ & 98.852 & 99.387 & $\overline{999.286}$ & 99.397 & 99.316 & 97.659 & 97.981 & 97.961 & $\overline{998.049}$ & 97.948 \\
\hline $4 \%$ & 98.988 & 99.358 & 99.309 & 99.370 & 99.301 & 98.242 & 98.541 & 98.552 & 98.705 & 98.649 \\
\hline $8 \%$ & 99.085 & 99.368 & 99.288 & 99.422 & 99.224 & 98.146 & 98.291 & 98.293 & 98.382 & 98.184 \\
\hline $12 \%$ & 99.155 & 99.399 & 99.329 & 99.431 & 99.193 & 97.971 & 97.807 & 97.789 & 98.046 & 97.814 \\
\hline $16 \%$ & 99.121 & 99.371 & 99.284 & 99.399 & 99.043 & 98.036 & 97.986 & 97.968 & 98.222 & 98.005 \\
\hline $20 \%$ & 99.115 & 99.346 & 99.217 & 99.403 & 98.924 & 97.962 & 98.063 & 98.079 & 98.170 & 97.945 \\
\hline $24 \%$ & 99.152 & 99.350 & 99.252 & 99.412 & 98.998 & 97.806 & 97.960 & 97.940 & 98.149 & 97.768 \\
\hline $28 \%$ & 99.147 & 99.344 & 99.210 & 99.414 & 98.763 & 97.772 & 97.884 & 97.838 & 98.250 & 97.794 \\
\hline $32 \%$ & 99.166 & 99.349 & 99.224 & 99.437 & 98.817 & 97.792 & 97.844 & 97.750 & 98.310 & 97.828 \\
\hline $36 \%$ & 99.256 & 99.419 & 99.278 & 99.489 & 98.809 & 97.808 & 97.847 & 97.769 & 98.189 & 97.749 \\
\hline $40 \%$ & 99.188 & 99.355 & 99.205 & 99.430 & 98.398 & 97.554 & 97.811 & 97.788 & 98.120 & 97.424 \\
\hline $44 \%$ & 99.106 & 99.301 & 99.167 & 99.392 & 98.727 & 97.599 & 97.940 & 97.935 & 98.224 & 97.635 \\
\hline $48 \%$ & 99.164 & 99.345 & 99.184 & 99.419 & 98.511 & 97.681 & 97.910 & 97.839 & 98.143 & 97.259 \\
\hline $52 \%$ & 99.133 & 99.341 & 99.165 & 99.428 & 98.640 & 97.655 & 98.036 & 97.996 & 98.430 & 97.504 \\
\hline $56 \%$ & 99.129 & 99.328 & 99.088 & 99.410 & 98.529 & 97.647 & 98.009 & 97.905 & 98.364 & 97.471 \\
\hline $60 \%$ & 99.062 & 99.264 & 99.020 & 99.356 & 98.163 & 97.453 & 97.846 & 97.595 & 97.909 & 97.238 \\
\hline $64 \%$ & 98.907 & 99.171 & 98.887 & 99.302 & 98.379 & 97.139 & 97.525 & 97.379 & 98.037 & 97.544 \\
\hline $68 \%$ & 98.792 & 99.120 & 98.624 & 99.337 & 98.022 & 97.152 & 97.654 & 97.472 & 97.948 & 97.065 \\
\hline $72 \%$ & 98.618 & 98.980 & 98.377 & 99.262 & 97.851 & 97.291 & 97.610 & 97.165 & 98.012 & 96.877 \\
\hline $76 \%$ & 98.150 & 98.694 & 98.052 & 99.091 & 97.331 & 96.439 & 97.159 & 96.767 & 97.828 & 96.400 \\
\hline $80 \%$ & 97.663 & 98.352 & 97.428 & 98.842 & 96.898 & 96.199 & 97.057 & 96.393 & 97.573 & 95.790 \\
\hline $84 \%$ & 96.953 & 97.760 & 96.104 & 98.576 & 96.211 & 95.478 & 96.538 & 95.407 & 97.324 & 95.066 \\
\hline $88 \%$ & 96.563 & 97.287 & 95.385 & 98.422 & 95.765 & 95.564 & 96.459 & 94.891 & 97.697 & 94.976 \\
\hline $92 \%$ & 96.306 & 97.175 & 95.482 & 98.182 & 95.356 & 95.588 & 96.577 & 95.155 & 97.478 & 94.695 \\
\hline
\end{tabular}

as well as Figs. 10 and 11. The fuzzy sets in these rules are semantically interpretable as very low, low, medium, high, very high.

The Wang-Mendel method (WM) was applied in order to generate the rules of this type from the dataset. However, these rules can be modified by a learning procedure to achieve better performance of the system; see the work of Rutkowski et al. (2018b), where a neuro-fuzzy recommender is presented. It should be emphasized that there is a trade-off between the interpretability and accuracy of the system. The learning procedure changes the fuzzy sets, making them less interpretable. Therefore, in this paper, we do not want to modify the rules in this way. Instead, the weights are assigned to fuzzy IF-THEN rules.

Applying the weights causes modification of decision boundaries in the attribute space, as Ishibuchi and Nakashima (2001) explained in their paper. Thus, the rules are better adjusted to the data, resulting in better performance of the system. It is worth adding that Nauck and Kruse (1998) show that by introducing the weights we obtain the same effect as by modification of fuzzy sets in the rules. As Ishibuchi and Nakashima (2001) illustrated, the rule weights also change the decision boundaries to achieve better performance of a classifier.

It should be emphasized that, when a rule is missing in the Wang-Mendel table, the decision boundaries are also different, as Ishibuchi and Nakashima (2001) showed.
Thus, removing a rule from the rule base changes the performance of the system. Therefore, in Algorithm 1 proposed in this paper, the recommender is evaluated after the removal of the least beneficial fuzzy rule.

With regard to Algorithm 1, several variants of the system are considered: WM, WO-C1, WR-C1, WO-C2, WR-C2 (see Tables 144). The first one (WM) is a system with the rules generated by the WM method, without the rule weights. Analyzing the results included in these tables, we observe that the systems with the weights perform better than the WM recommender.

Compared the different variants of the system with the weights, we see, as concluded in Section 4.2, that, generally, WO-C2 gives better results. This is a system with optimized weights (and not rounded) in the case when the weights have not been reset. This means that the procedure of reducing the rules always concerns the same system (not changed by resetting the values of the weights).

It also seems obvious that better performance is obtained for the system with optimized weights and not rounded values. However, this is also a compromise between accuracy and interpretability.

The approach proposed in this paper, as mentioned in Section 11 is enhanced by the criteria (AIC, FPE, Szwarz) for evaluating an optimal balance in terms of the recommender's accuracy and interpretability.

In future work, it would be worth applying other 
Table 5. AIC, FPE and Schwarz criteria for different numbers of fuzzy rules ( $p$ is equal to the number of fuzzy rules and thus the number of weights, $Q$ stands for the RMSE of learning samples for the WO method).

\begin{tabular}{|c|c|c|c|c|c|c|c|c|}
\hline \multirow[b]{2}{*}{$p$} & \multicolumn{4}{|c|}{ WO-C1 } & \multicolumn{4}{|c|}{ WO-C2 } \\
\hline & $\bar{Q}$ & AIC & FPE & Szwarz & $\bar{Q}$ & AIC & FPE & Szwarz \\
\hline 36 & $\overline{\overline{0.209}}$ & $\overline{-7.927}$ & $\overline{\overline{0.337}}$ & 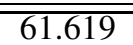 & $\overline{0.198}$ & -10.650 & $\overline{\overline{0.319}}$ & $\overline{58.896}$ \\
\hline 35 & 0.204 & -11.108 & 0.325 & 56.506 & 0.193 & -14.007 & 0.307 & 53.607 \\
\hline 34 & 0.199 & -14.378 & 0.312 & 51.304 & 0.188 & -17.250 & 0.295 & 48.432 \\
\hline 33 & 0.193 & -17.810 & 0.300 & 45.940 & 0.182 & -21.027 & 0.281 & 42.723 \\
\hline 32 & 0.186 & -21.873 & 0.284 & 39.946 & 0.174 & -25.159 & 0.266 & 36.659 \\
\hline 31 & 0.181 & -25.184 & 0.273 & 34.702 & 0.169 & -28.662 & 0.255 & 31.225 \\
\hline 30 & 0.176 & -28.475 & 0.263 & 29.480 & 0.165 & -31.851 & 0.246 & 26.104 \\
\hline 29 & 0.172 & -31.919 & 0.252 & 24.104 & 0.160 & -35.370 & 0.235 & 20.653 \\
\hline 28 & 0.169 & -34.766 & 0.244 & 19.325 & 0.157 & -38.293 & 0.228 & 15.798 \\
\hline 27 & 0.166 & -37.447 & 0.238 & 14.712 & 0.155 & -41.215 & 0.221 & 10.944 \\
\hline 26 & 0.164 & -40.245 & 0.231 & 9.982 & 0.152 & -44.120 & 0.214 & 6.108 \\
\hline 25 & 0.160 & -43.477 & 0.222 & 4.818 & 0.148 & -47.511 & 0.206 & 0.784 \\
\hline 24 & 0.156 & -46.688 & 0.214 & -0.324 & 0.144 & -51.000 & 0.197 & -4.636 \\
\hline 23 & 0.154 & -49.434 & 0.208 & -5.002 & 0.141 & -53.811 & 0.191 & -9.379 \\
\hline 22 & 0.152 & -52.208 & 0.203 & -9.707 & 0.138 & -56.835 & 0.185 & -14.335 \\
\hline 21 & 0.148 & -55.310 & 0.196 & -14.742 & 0.135 & -59.978 & 0.178 & -19.410 \\
\hline 20 & 0.146 & -58.138 & 0.190 & -19.501 & 0.133 & -62.931 & 0.173 & -24.295 \\
\hline 19 & 0.144 & -60.752 & 0.185 & -24.047 & 0.131 & -65.756 & 0.168 & -29.051 \\
\hline 18 & 0.142 & -63.454 & 0.180 & -28.681 & 0.128 & -68.664 & 0.163 & -33.891 \\
\hline 17 & 0.141 & -65.943 & 0.176 & -33.102 & 0.126 & -71.619 & 0.158 & -38.778 \\
\hline 16 & 0.141 & -67.811 & 0.174 & -36.902 & 0.125 & -73.909 & 0.155 & -43.000 \\
\hline 15 & 0.142 & -69.412 & 0.173 & -40.435 & 0.125 & -75.875 & 0.153 & -46.898 \\
\hline 14 & 0.144 & -70.893 & 0.173 & -43.848 & 0.126 & -77.662 & 0.151 & -50.617 \\
\hline 13 & 0.146 & -71.969 & 0.174 & -46.855 & 0.127 & -79.091 & 0.151 & -53.977 \\
\hline 12 & 0.150 & -72.770 & 0.175 & -49.588 & 0.130 & -80.194 & 0.152 & -57.012 \\
\hline 11 & 0.155 & -73.184 & 0.179 & -51.934 & 0.133 & -80.806 & 0.154 & -59.556 \\
\hline 10 & 0.162 & -72.892 & 0.184 & -53.574 & 0.139 & -80.701 & 0.158 & -61.383 \\
\hline 9 & 0.172 & -71.723 & 0.194 & -54.336 & 0.148 & -79.608 & 0.166 & -62.221 \\
\hline 8 & 0.187 & -69.512 & 0.208 & -54.057 & 0.161 & -77.127 & 0.179 & -61.673 \\
\hline 7 & 0.207 & -66.410 & 0.226 & -52.887 & 0.179 & -73.725 & 0.196 & -60.203 \\
\hline 6 & 0.234 & -62.152 & 0.253 & -50.561 & 0.202 & -69.562 & 0.219 & -57.971 \\
\hline 5 & 0.272 & -56.470 & 0.290 & -46.811 & 0.235 & -63.937 & 0.250 & -54.278 \\
\hline 4 & 0.319 & -50.329 & 0.336 & -42.601 & 0.276 & -57.706 & 0.291 & -49.979 \\
\hline
\end{tabular}

rule-based techniques (see, e.g., Bologna and Hayashi, 2017; Liu et al., 2017) to design explainable recommender systems.

\section{Acknowledgment}

This research was supported by the Polish National Science Center under the grant 2015/19/B/ST6/03179.

\section{References}

Alvarez-Estevez, D., Moret-Bonillo, V. (2018). Revisiting the Wang-Mendel algorithm for fuzzy classification, Expert Systems 35(4): 35:312268.

Bologna, G. and Hayashi, Y. (2017). Characterization of symbolic rules embedded in deep DIMLP networks: A challenge to transparency of deep learning, Journal of Artificial Intelligence and Soft Computing Research 7(4): 265-286.
Cpalka, K. (2017). Design of Interpretable Fuzzy Systems, Studies in Computational Intelligence 684, Springer Verlag, Cham.

Harper, F.M. and Konstan, J.A. (2015). The MovieLens datasets: History and context, ACM Transactions on Interactive Intelligent Systems 5(4):19:1-19:19.

Ishibuchi H. and T. Nakashima (2001). Effect of rule weights in fuzzy rule-based classification systems, IEEE Transactions on Fuzzy Systems 9(4): 506-515.

Ishibuchi H. and T. Yamamoto (2005). Rule weight specification in fuzzy rule-based classification systems, IEEE Transactions on Fuzzy Systems 13(4): 428-435.

Jin, Y. (2000). Fuzzy modeling of high-dimensional systems: Complexity reduction and interpretability improvement, IEEE Transactions on Fuzzy Systems 8(2): 212-221.

Kuncheva, L. (2000). Fuzzy Classifier Design, Studies in Fuzziness and Soft Computing, Vol. 49, Springer Verlag, New York, NY. 

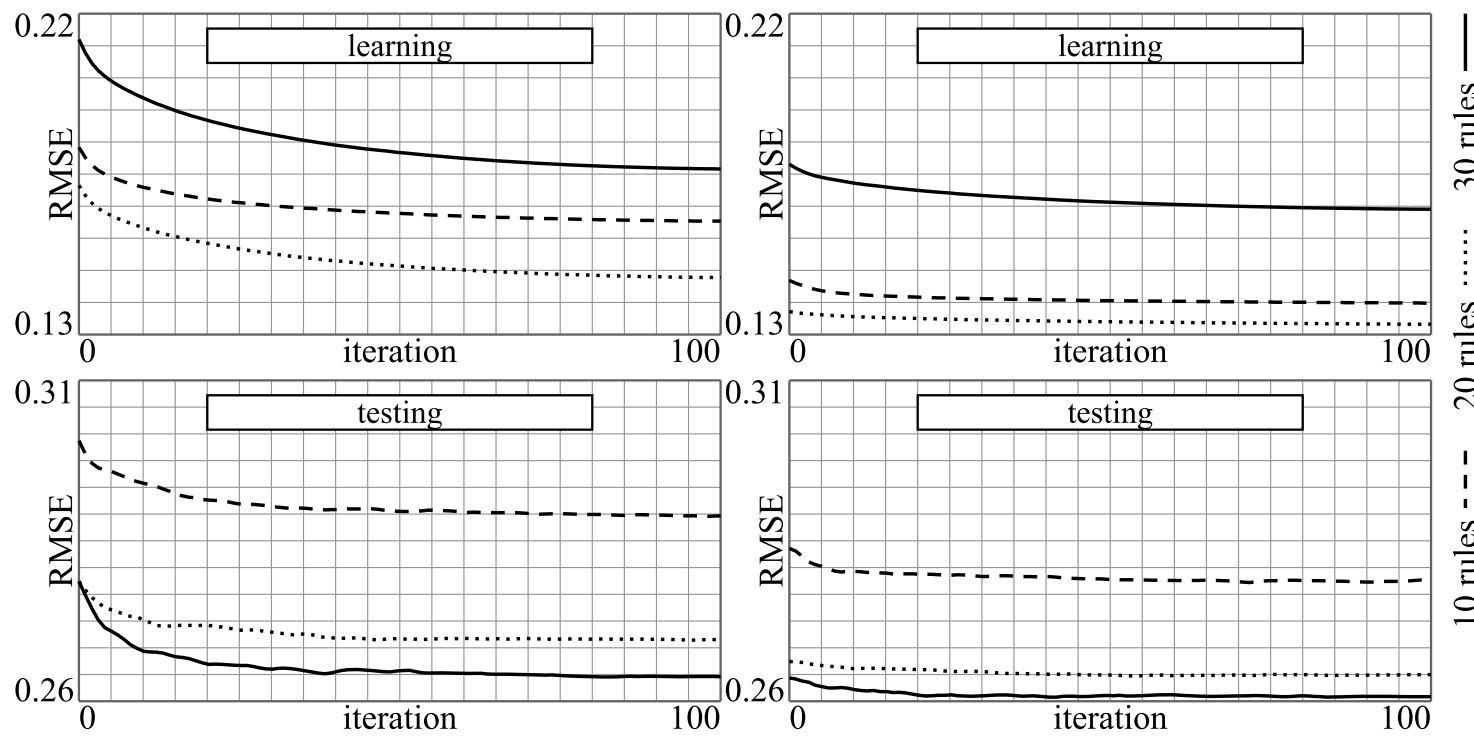

(a) WO-C1

(b) WO-C2

Fig. 9. Optimization of weights for different numbers of fuzzy rules.
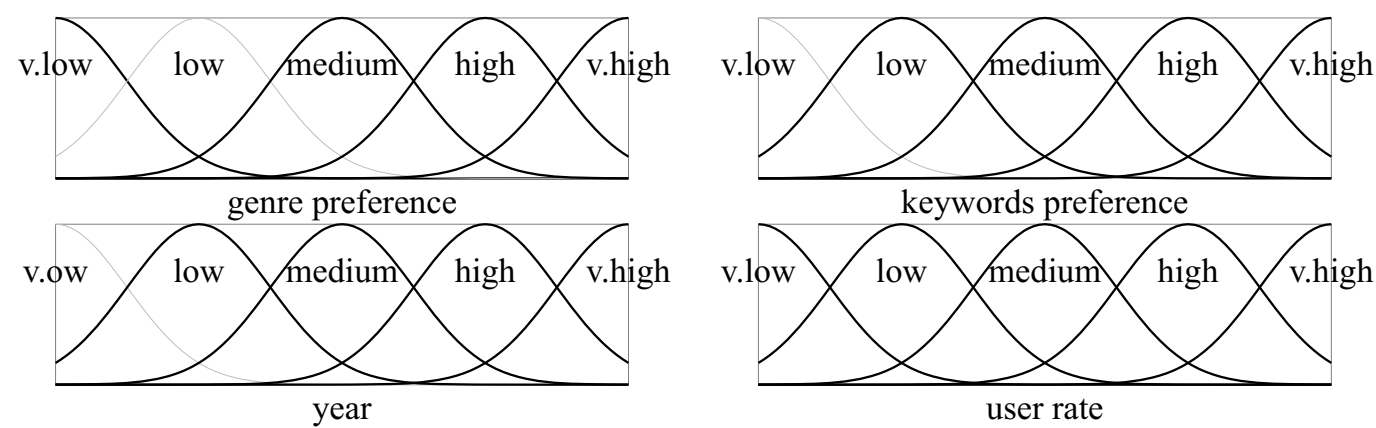

Fig. 10. Fuzzy sets in fuzzy rules from Table 7.

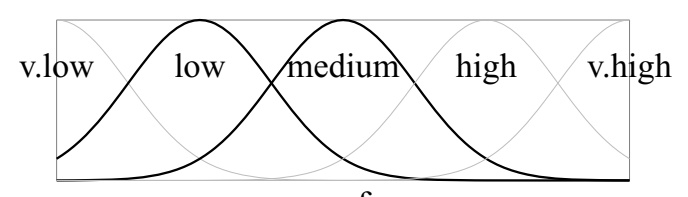

genre preference

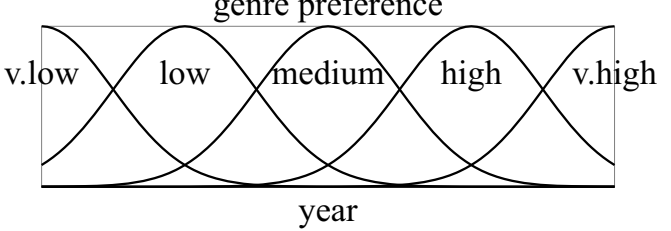

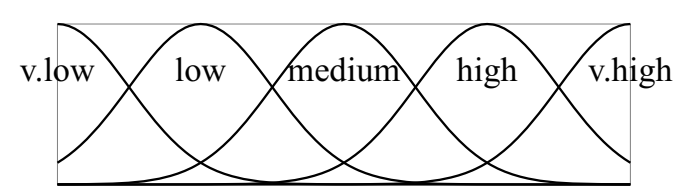

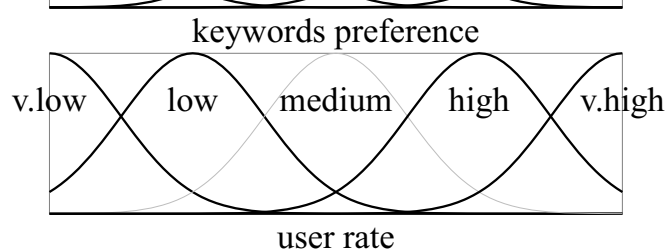

Fig. 11. Fuzzy sets in fuzzy rules from Table 8. 
Table 6. AIC, FPE and Schwarz criteria for different numbers of fuzzy rules ( $p$ is equal to number of fuzzy rules and thus the number of weights, $Q$ stands for the RMSE of testing samples for the WO method).

\begin{tabular}{|c|c|c|c|c|c|c|c|c|}
\hline \multirow[b]{2}{*}{$p$} & \multicolumn{4}{|c|}{ WO-C1 } & \multicolumn{4}{|c|}{ WO-C2 } \\
\hline & $\mathrm{Q}$ & $\overline{\mathrm{AIC}}$ & FPE & Szwarz & $\bar{Q}$ & AIC & FPE & Szwarz \\
\hline 36 & 0.261 & 3.529 & 0.422 & 73.075 & 0.258 & 2.977 & 0.417 & $\overline{72.523}$ \\
\hline 35 & 0.248 & -1.097 & 0.395 & 66.517 & 0.248 & -1.205 & 0.394 & 66.409 \\
\hline 34 & 0.244 & -3.937 & 0.383 & 61.745 & 0.245 & -3.678 & 0.385 & 62.004 \\
\hline 33 & 0.252 & -4.352 & 0.390 & 59.398 & 0.250 & -4.720 & 0.387 & 59.031 \\
\hline 32 & 0.261 & -4.448 & 0.399 & 57.370 & 0.259 & -4.969 & 0.395 & 56.850 \\
\hline 31 & 0.260 & -6.671 & 0.392 & 53.215 & 0.261 & -6.593 & 0.393 & 53.293 \\
\hline 30 & 0.264 & -7.955 & 0.393 & 50.000 & 0.261 & -8.564 & 0.388 & 49.391 \\
\hline 29 & 0.261 & -10.490 & 0.383 & 45.533 & 0.257 & -11.338 & 0.377 & 44.685 \\
\hline 28 & 0.260 & -12.640 & 0.377 & 41.4 & 0.258 & -13.185 & 0.373 & 40.906 \\
\hline 27 & 0.264 & -13.922 & 0.377 & 38.2 & .260 & -14.710 & 0.371 & 37.449 \\
\hline 26 & 0.264 & -15.828 & 0.373 & 34.399 & 0.260 & -16.672 & 0.367 & 33.556 \\
\hline 25 & 0.265 & -17.701 & 0.369 & 30.595 & 0.263 & -18.119 & 0.366 & 30.176 \\
\hline 24 & 0.268 & -19.075 & 0.368 & 27.2 & 0.264 & -19.891 & 0.362 & 26.473 \\
\hline 23 & 0.273 & -20.241 & 0.369 & 24.191 & 0.269 & -20.931 & 0.364 & 23.501 \\
\hline 22 & 0.271 & -22.547 & 0.362 & 19. & .266 & -23.616 & 0.355 & 18.884 \\
\hline 21 & 0.269 & -25.057 & 0.354 & 15. & 0.264 & -25.933 & 0.348 & 14.636 \\
\hline 20 & 0.270 & -26.846 & 0.351 & 11.791 & 0.264 & -27.898 & 0.344 & 10.739 \\
\hline 19 & 0.271 & -28.534 & 0.348 & 8.171 & 0.265 & -29.773 & 0.340 & 6.932 \\
\hline 18 & 0.274 & -29.968 & 0.347 & 4.8 & 0.269 & -30.939 & 0.341 & 3.834 \\
\hline 17 & 0.274 & -32.094 & 0.342 & 0.7 & 0.268 & -33.112 & 0.335 & -0.271 \\
\hline 16 & 0.277 & -33.530 & 0.341 & -2.6 & 0.269 & -35.015 & 0.332 & -4.106 \\
\hline 15 & 0.278 & -35.233 & 0.339 & -6.255 & 0.270 & -36.817 & 0.328 & -7.840 \\
\hline 14 & 0.281 & -36.777 & 0.337 & -9.731 & 0.272 & -38.441 & 0.327 & -11.396 \\
\hline 13 & 0.282 & -38.590 & 0.334 & -13.476 & 0.274 & -40.013 & 0.325 & -14.899 \\
\hline 12 & 0.281 & -40.805 & 0.328 & -17.623 & 0.275 & -41.839 & 0.322 & -18.657 \\
\hline 11 & 0.286 & -41.903 & & -20.6 & 0.278 & -43.284 & 0.321 & -22.034 \\
\hline 10 & 0.289 & -43.327 & 0.329 & -24.0 & 0.279 & -45.068 & 0.318 & -25.750 \\
\hline 9 & 0.292 & -44.838 & 0.328 & -27.4 & 0.277 & -47.3 & 0.312 & -30.001 \\
\hline 8 & 0.300 & -45.332 & 0.334 & -29.878 & 0.281 & -48.673 & 0.312 & -33.218 \\
\hline 7 & 0.315 & -44.962 & 0.345 & -31.440 & 0.288 & -49.446 & 0.316 & -35.923 \\
\hline 6 & 0.329 & -44.724 & 0.356 & -33.133 & 0.299 & -49.577 & 0.323 & -37.986 \\
\hline 5 & 0.351 & -43.439 & & -33.7 & 0.311 & -49.639 & 0.332 & -39.980 \\
\hline 4 & 0.373 & -42.362 & 0.393 & -34.635 & 0.327 & -49.043 & 0.344 & -41.316 \\
\hline
\end{tabular}

Liu, H., Gegov, A. and Cocea, M. (2017). Rule based networks: An efficient and interpretable representation of computational models, Journal of Artificial Intelligence and Soft Computing Research 7(2): 111-123.

Lops, P., Gemmis, M. and Semeraro, G. (2011). Content-based recommender systems: State of the art and trends, in F. Ricci et al. (Eds), Recommender Systems Handbook, Springer, New York, NY, pp.73-105.

Nauck, D. and R. Kruse (1998). How the learning of rule weights affects the interpretability of fuzzy systems, IEEE International Conference on Fuzzy Systems 1998 (FUZZ-IEEE'98), Ancorage, AK, USA, pp.1235-1240.

Portugal, I., Paulo S.C. Alencar and Cowan, D.D. (2018). The use of machine learning algorithms in recommender systems: A systematic review, Expert System Applications 97: 205-227.

Prasad, M., Liu, Y.-T., Li, D.-L., Lin, C.-T., Shah, R.R. and Kaiwartya, O.P. (2017). A new mechanism for data visualization with TSK-type preprocessed collaborative fuzzy rule based system, Journal of Artificial Intelligence and Soft Computing Research 7(1): 33-46.

Riid, A. and Preden, J.-S. (2017). Design of fuzzy rule-based classifiers through granulation and consolidation, Journal of Artificial Intelligence and Soft Computing Research 7(2): 137-147.

Rutkowska, D. (2002). Neuro-Fuzzy Architectures and Hybrid Learning, Studies in Fuzziness and Soft Computing, Springer Verlag, New York, NY.

Rutkowski, L. (2004). Flexible Neuro-Fuzzy Systems: Structures, Learning and Performance Evaluation, Kluwer Academic Publishers, Boston, MA/Dordrecht/London.

Rutkowski, L. (2008). Computational Intelligence: Methods and Techniques, Springer, Berlin.

Rutkowski, T., Romanowski, J., Woldan, P., Staszewski, P. and Nielek, R. (2018). Towards interpretability of the movie recommender based on a neuro-fuzzy approach, 17th International Conference on Artificial Intelligence and Soft Computing, ICAISC 2018, Zakopane, Poland, pp. 752-762. 
Table 7. Examples of fuzzy rules for the user with id $=7$ and optimal reduction of fuzzy rules considering the obtained accuracy.

\begin{tabular}{|c|c|c|c|c|c|c|c|}
\hline \multirow[b]{2}{*}{$k$} & \multicolumn{3}{|c|}{ IF } & THEN & \multicolumn{3}{|c|}{ rule weight } \\
\hline & $\begin{array}{l}\text { genre } \\
\text { pref. is }\end{array}$ & $\begin{array}{l}\text { year } \\
\text { is }\end{array}$ & $\begin{array}{l}\text { keywords } \\
\text { pref. is }\end{array}$ & $\begin{array}{c}\text { user rate } \\
\text { is }\end{array}$ & WM & WO-C2 & WR-C2 \\
\hline 1 & high & v.high & medium & medium & 0.500 & 0.543 & 0.500 \\
\hline 2 & high & high & v.high & v.high & 0.500 & 0.076 & 0.100 \\
\hline 3 & v.high & v.high & v.high & v.high & 0.500 & 0.917 & 0.900 \\
\hline 4 & high & medium & v.high & v.high & 0.500 & 0.604 & 0.600 \\
\hline 5 & high & medium & high & high & 0.500 & 0.228 & 0.200 \\
\hline 6 & high & low & v.high & v.high & 0.500 & 0.620 & 0.600 \\
\hline 7 & medium & v.high & low & v.low & 0.500 & 0.591 & 0.600 \\
\hline 8 & v.high & v.high & high & high & 0.500 & 0.465 & 0.500 \\
\hline 9 & high & v.high & low & v.low & 0.500 & 0.580 & 0.600 \\
\hline 10 & high & low & medium & low & 0.500 & 0.443 & 0.400 \\
\hline 11 & high & medium & medium & medium & 0.500 & 0.296 & 0.300 \\
\hline 12 & high & high & low & v.low & 0.500 & 0.149 & 0.100 \\
\hline 13 & high & low & high & high & 0.500 & 0.516 & 0.500 \\
\hline 14 & medium & v.high & v.high & v.high & 0.500 & 1.000 & 1.000 \\
\hline 15 & v.low & v.high & low & low & 0.500 & 0.642 & 0.600 \\
\hline 16 & medium & v.high & medium & medium & 0.500 & 0.345 & 0.300 \\
\hline & \multirow{6}{*}{\multicolumn{2}{|c|}{ system errors }} & \multirow{2}{*}{ RMSE } & learning & 0.247 & 0.210 & 0.212 \\
\hline & & & & testing & 0.344 & 0.330 & 0.328 \\
\hline & & & \multirow{2}{*}{$\mathrm{ACC}$} & learning & 75.1 & 82.0 & 80.1 \\
\hline & & & & testing & 57.1 & 65.8 & 61.9 \\
\hline & & & \multirow{2}{*}{ YES/NO } & learning & 99.4 & 99.4 & 99.4 \\
\hline & & & & testing & 100.0 & 100.0 & 100.0 \\
\hline
\end{tabular}

Table 8. Examples of fuzzy rules for the user with id $=2$ and optimal reduction of fuzzy rules considering the AIC, FPE and Schwarz criteria.

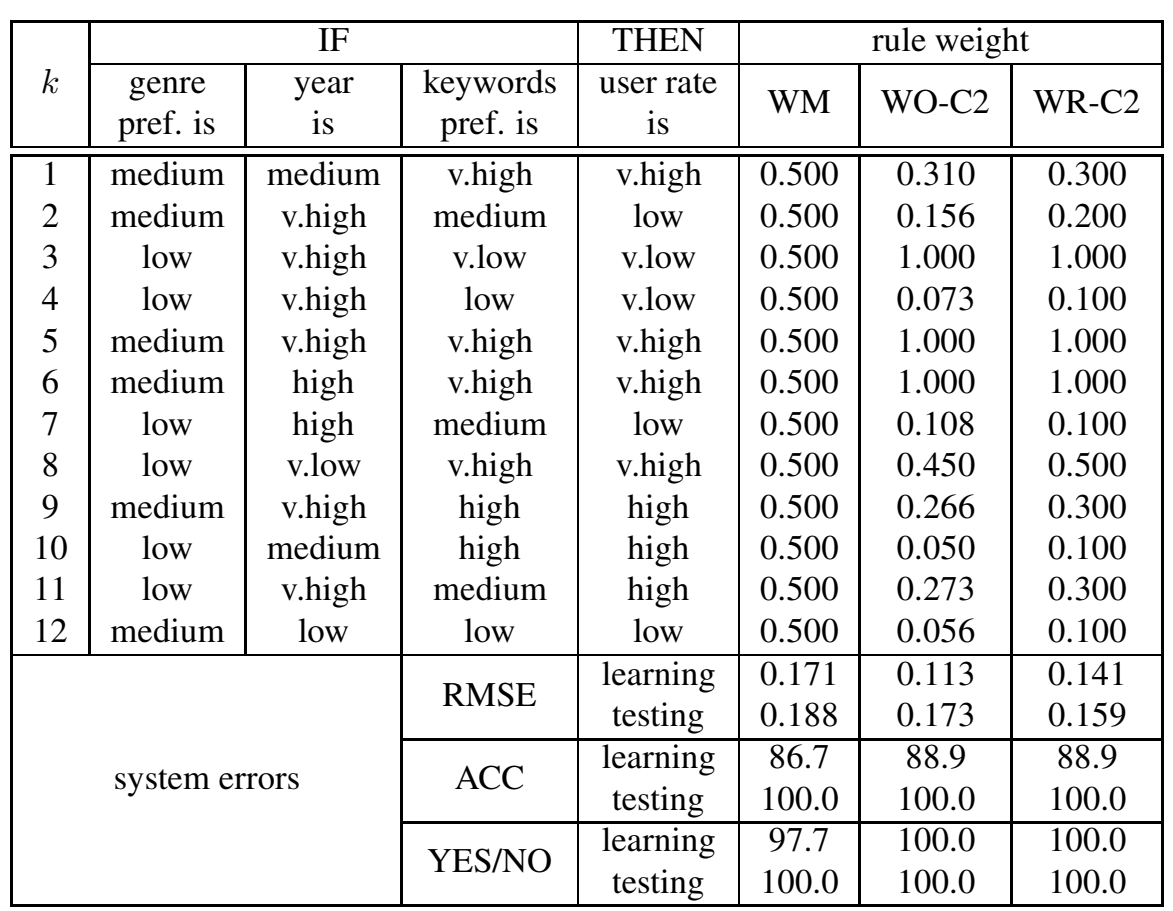


Rutkowski, T., Romanowski, J., Woldan, P., Staszewski, P., Nielek, R. and Rutkowski, L. (2018). A content-based recommendation system using neuro-fuzzy approach, 2018 IEEE International Conference on Fuzzy Systems (FUZZ-IEEE 2018), Piscataway, NJ, USA, pp. 1-8, DOI:10.1109/FUZZ-IEEE.2018.8491543.

Simiński, K. (2010). Rule weights in a neuro-fuzzy system with a hierarchical domain partition, International Journal of Applied Mathematics and Computer Science 20(2): 337-347, DOI: 10.2478/v10006-010-0025-3.

Söderström, T. and Stoica, P. (1989). System Identification, Prentice Hall International, Upper Saddle River, NJ.

Wang, L.-X. and Mendel J.M. (1992). Generating fuzzy rules by learning from examples, IEEE Transactions on Systems, Man, and Cybernetics: Systems 22(6): 1414-1427.

Wei, J., He, J., Chen, K., Zhou, Y. and Tang, Z. (2017). Collaborative filtering and deep learning based recommendation system for cold start items, Expert Systems with Applications 69: 29-39.

Zhang, S., Yao L., Sun A. and Y. Tay (2018). Deep learning based recommender system: A survey and new perspectives, ACM Computing Surveys 52(1), Article No. 5.

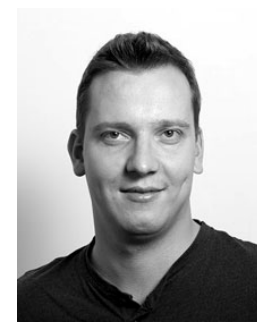

Tomasz Rutkowski received his MSc degree in computer engineering from the Polish-Japanese Academy of Information Technology and an MBA degree from Kozminski University in Warsaw, Poland. He is currently working on his $\mathrm{PhD}$ in computer science and management. His research interests concern AI explainability, transparency and the human-in-the-loop approach. He is also a co-CEO at Senfino, an explainable AI company based in New York City.

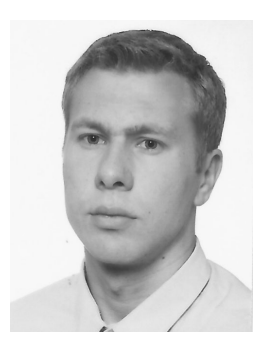

Krystian Łapa is an associate professor in the Institute of Computational Intelligence at the Czestochowa University of Technology, where he received his $\mathrm{PhD}$ degree in 2015. His research interests include fuzzy systems, interpretability, population-based algorithms, nonlinear modelling and, in the broad sense, computational intelligence.

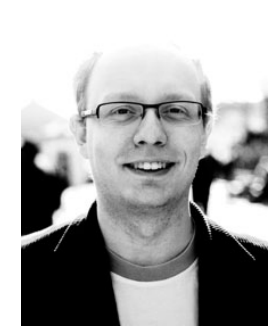

Radosław Nielek is an associate professor at the Polish-Japanese Academy of Information Technology, where he has received his $\mathrm{PhD}$ degree. His research interests include co-design of ICTs for the elderly focused on improving life quality, web credibility and social informatics. He has published over 70 papers on web mining, social informatics and social simulation.

Received: 5 March 2019

Revised: 13 April 2019

Accepted: 29 April 2019 